ANNALES

POLONICI MATHEMATICI

$89.1(2006)$

\title{
Controllability results for first and second order evolution inclusions with nonlocal conditions
}

\author{
by L. Górniewicz (Toruń), S. K. Ntouyas (Ioannina) \\ and D. O'REgan (Galway)
}

Abstract. We prove controllability results for first and second order semilinear differential inclusions in Banach spaces with nonlocal conditions.

1. Introduction. In this paper, we give some controllability results for first and second order semilinear differential inclusions in a real Banach space, with nonlocal conditions.

In Section 3 we study controllability for first order semilinear nonlocal initial value problems of the form

$$
\begin{gathered}
y^{\prime}(t) \in A y(t)+F(t, y(t))+\mathcal{B} u(t), \quad t \in J:=[0, b], \\
y(0)+f(y)=y_{0},
\end{gathered}
$$

where $F: J \times E \rightarrow \mathcal{P}(E)$ is a multivalued map $(\mathcal{P}(E)$ is the family of all nonempty subsets of $E), A: D(A) \subset E \rightarrow E$ is the infinitesimal generator of a semigroup $\{T(t): t \geq 0\}, y_{0} \in E, f: C(J, E) \rightarrow E$ is continuous and $E$ a real separable Banach space with norm $|\cdot|$. Also the control function $u(\cdot)$ is in $L^{2}(J, U)$, where $U$ is a Banach space. Finally, $\mathcal{B}$ is a bounded linear operator from $U$ to $E$.

A special case of the nonlocal condition is studied in Section 4. In Section 5 we consider the problem (1.1)-(1.2) where $A: D(A) \subset E \rightarrow E$ is a nondensely defined closed linear operator.

In Section 6 we study controllability for initial value problems of the form

$$
\begin{gathered}
y^{\prime \prime}(t) \in A y(t)+F(t, y(t))+\mathcal{B} u(t), \quad t \in J, \\
y(0)+f(y)=y_{0}, \quad y^{\prime}(0)+f_{1}(y)=\eta,
\end{gathered}
$$

2000 Mathematics Subject Classification: Primary 93B05.

Key words and phrases: semilinear differential inclusions, controllability, nonlocal conditions, semigroup, cosine functions, integrated semigroups, fixed point, nonlinear alternative. 
where $A$ is the infinitesimal generator of a family of cosine operators $\{C(t)$ : $t \geq 0\}, \eta \in E$ and $F, y_{0}, f, \mathcal{B}, u$ are as in problem (1.1)-(1.2) and $f_{1}$ : $C(J, E) \rightarrow E$ is continuous.

The study of nonlocal conditions for evolution equations was initiated by Byszewski. We refer the reader to [5] and the references therein for a motivation. The nonlocal conditions can be applied in physics and are more natural than the classical initial condition $y(0)=y_{0}$. For example, $f(y)$ may be given by

$$
f(y)=\sum_{i=1}^{p} c_{i} y\left(t_{i}\right),
$$

where $c_{i}, i=1, \ldots, p$, are given constants and $0<t_{1}<\cdots<t_{p} \leq b$.

Recently in [11] we studied existence results for the problems (1.1)-(1.2) and (1.3)-(1.4) where $\mathcal{B}=0$, by assuming the existence of maximal solutions to appropriate problems. Controllability results for these problems (with $f_{1}=0$ ) were given in [3]. However, the results in [3] are incorrect since the constants $M_{0}$ on page 39 and $\bar{M}_{0}$ on page 103 depend on the solution. Here we correct these results using fixed point methods, in particular the nonlinear alternative for single-valued and Kakutani maps, Kakutani's fixed point theorem and a selection theorem for lower semicontinuous maps.

2. Preliminaries. In this section, we introduce notations, definitions, and preliminary facts that are used throughout this paper.

Let $X$ be a subset of a metric linear space. We consider the following subsets of $\mathcal{P}(X)=\{Y \subset X: Y \neq \emptyset\}$ :

$$
\begin{aligned}
\mathcal{P}_{\mathrm{cl}}(X) & =\{Y \in \mathcal{P}(X): Y \text { closed }\}, \\
\mathcal{P}_{\mathrm{b}}(X) & =\{Y \in \mathcal{P}(X): Y \text { bounded }\}, \\
\mathcal{P}_{\mathrm{c}}(X) & =\{Y \in \mathcal{P}(X): Y \text { convex }\}, \\
\mathcal{P}_{\mathrm{cp}}(X) & =\{Y \in \mathcal{P}(X): Y \text { compact }\}, \\
\mathcal{P}_{\mathrm{c}, \mathrm{cp}}(X) & =\mathcal{P}_{\mathrm{c}}(X) \cap \mathcal{P}_{\mathrm{cp}}(X) \text { etc. }
\end{aligned}
$$

A multivalued map $G: X \rightarrow \mathcal{P}(X)$ is convex-valued (resp. closed-valued) if $G(x)$ is convex (resp. closed) for all $x \in X$. $G$ is bounded on bounded sets if $G(\mathcal{B}):=\bigcup_{x \in \mathcal{B}} G(x)$ is bounded in $X$ for all $\mathcal{B} \in \mathcal{P}_{\mathrm{b}}(X)$.

$G$ is called upper semicontinuous (u.s.c.) on $X$ if for each $x_{0} \in X$ the set $G\left(x_{0}\right)$ is a nonempty, closed subset of $X$, and for each open subset $\mathcal{U}$ of $X$ containing $G\left(x_{0}\right)$, there exists an open neighborhood $\mathcal{V}$ of $x_{0}$ such that $G(\mathcal{V}) \subseteq \mathcal{U}$

$G$ is said to be completely continuous if $G(\mathcal{B})$ is relatively compact for every $\mathcal{B} \in \mathcal{P}_{\mathrm{b}}(X)$. If $G$ is completely continuous with nonempty compact values, then $G$ is u.s.c. if and only if $G$ has closed graph (i.e. $x_{n} \rightarrow x_{*}$, 
$y_{n} \rightarrow y_{*}, y_{n} \in G\left(x_{n}\right)$ imply $\left.y_{*} \in G\left(x_{*}\right)\right)$. $G$ has a fixed point if there is $x \in X$ such that $x \in G(x)$. The fixed point set of the multivalued operator $G$ will be denoted by Fix $G$.

A multivalued map $N: J \rightarrow \mathcal{P}_{\mathrm{cl}}(E)$ is said to be measurable if for every $y \in E$, the function $t \mapsto d(y, N(t))=\inf \{|y-z|: z \in N(t)\}$ is measurable. For more details on multivalued maps see the books of Aubin and Cellina [2], Deimling [8], Górniewicz [10] and Hu and Papageorgiou [15].

Let $E$ be a Banach space and $B(E)$ be the Banach space of bounded linear operators on $E$.

Definition 2.1. A $C_{0}$ semigroup or a semigroup of class $\left(C_{0}\right)$ is a oneparameter family $\{T(t): t \geq 0\} \subset B(E)$ satisfying the conditions:

(i) $T(t) \circ T(s)=T(t+s)$ for $t, s \geq 0$,

(ii) $T(0)=I$ (the identity operator in $E$ ),

(iii) the map $t \mapsto T(t) x$ is strongly continuous for each $x \in E$, i.e.

$$
\lim _{t \rightarrow 0} T(t) x=x, \quad \forall x \in E .
$$

The semigroup is uniformly continuous if

$$
\lim _{t \rightarrow 0}\|T(t)-I\|=0 .
$$

We note that if a semigroup $T(t)$ is of class $\left(C_{0}\right)$, then we have the growth condition $\|T(t)\|_{B(E)} \leq M e^{\beta t}$ for $0 \leq t<\infty$, with some constants $M>0$ and $\beta \in \mathbb{R}$. If, in particular, $M=1$ and $\beta=0$, i.e. $\|T(t)\|_{B(E)} \leq 1$ for $t \geq 0$, then $T(t)$ is called a contraction $C_{0}$ semigroup.

Definition 2.2. Let $T(t)$ be a $C_{0}$ semigroup on $E$. The infinitesimal generator $A$ of $T(t)$ is the linear operator defined by

$$
A(x)=\lim _{h \rightarrow 0} \frac{T(h) x-x}{h} \quad \text { for } x \in D(A),
$$

where

$$
D(A)=\left\{x \in E: \lim _{h \rightarrow 0} \frac{T(h) x-x}{h} \text { exists in } E\right\} .
$$

Proposition 2.1. The infinitesimal generator $A$ is a closed linear and densely defined operator in $E$. If $x \in D(A)$, then $T(t) x$ is a $C^{1}$-map and

$$
\frac{d}{d t} T(t) x=A(T(t) x)=T(t) A(x) \quad \text { on }[0, \infty) .
$$

It is well known ([18]) that an operator $A$ generates a $C_{0}$ semigroup if

(i) $\overline{D(A)}=E$,

(ii) the Hille-Yosida condition holds, that is, there exist $M \geq 0$ and $\omega \in \mathbb{R}$ such that $(\omega, \infty) \subset \varrho(A), \sup \left\{(\lambda I-\omega)^{n}\left|(\lambda I-A)^{-n}\right|: \lambda>\omega\right.$, $n \in \mathbb{N}\} \leq M$,

where $\varrho(A)$ is the resolvent set of $A$ and $I$ is the identity operator. 
We say that a family $\{C(t): t \in \mathbb{R}\} \subset B(E)$ is a strongly continuous cosine family if

(i) $C(0)=I$,

(ii) $C(t+s)+C(t-s)=2 C(t) C(s)$ for all $s, t \in \mathbb{R}$,

(iii) the map $t \mapsto C(t) x$ is strongly continuous for each $x \in E$.

The strongly continuous sine family $\{S(t): t \in \mathbb{R}\}$, associated to a given strongly continuous cosine family $\{C(t): t \in \mathbb{R}\}$, is defined by

$$
S(t) x=\int_{0}^{t} C(s) x d s, \quad x \in E, t \in \mathbb{R} .
$$

The infinitesimal generator $A: E \rightarrow E$ of a cosine family $\{C(t): t \in \mathbb{R}\}$ is defined by

$$
A(x)=\left.\frac{d^{2}}{d t^{2}} C(t) x\right|_{t=0} .
$$

For more details on strongly continuous cosine and sine families, we refer the reader to the books of Goldstein [12], Heikkila and Lakshmikantham [14] and Fattorini [9], and the papers [20] and [21].

Proposition 2.2 ([20]). Let $C(t), t \in \mathbb{R}$, be a strongly continuous cosine family in E. Then:

(i) there exist constants $M_{1} \geq 1$ and $\omega \geq 0$ such that $\|C(t)\| \leq M_{1} e^{\omega|t|}$ for all $t \in \mathbb{R}$

(ii) $\left\|S\left(t_{1}\right)-S\left(t_{2}\right)\right\| \leq M_{1}\left|\int_{t_{2}}^{t_{1}} e^{\omega|s|} d s\right|$ for all $t_{1}, t_{2} \in \mathbb{R}$.

Definition 2.3. A multivalued map $F: J \times E \rightarrow \mathcal{P}_{\text {c,cp }}(E)$ is said to be $L^{1}$ - Carathéodory if:

(i) $t \mapsto F(t, u)$ is measurable for each $u \in E$;

(ii) $u \mapsto F(t, u)$ is upper semicontinuous on $E$ for almost all $t \in J$;

(iii) for each $\varrho>0$, there exists $h_{\varrho} \in L^{1}\left(J, \mathbb{R}_{+}\right)$such that

$$
\|F(t, u)\|:=\sup \{|v|: v \in F(t, u)\} \leq h_{\varrho}(t)
$$

for all $|u| \leq \varrho$ and for a.e. $t \in J$.

The considerations of this paper are based on the following alternatives ([13]).

Theorem 2.1 (Nonlinear alternative for single-valued maps). Let $E$ be a Banach space, $C$ a closed, convex subset of $E, U$ an open subset of $C$ and $0 \in U$. Suppose that $F: \bar{U} \rightarrow C$ is a continuous, compact map (that is, $F(\bar{U})$ is a relatively compact subset of $C$ ). Then either

(i) F has a fixed point in $\bar{U}$, or

(ii) there are $u \in \partial U$ (the boundary of $U$ in $C$ ) and $\lambda \in(0,1)$ with $u=\lambda F(u)$. 
Theorem 2.2 (Nonlinear alternative for Kakutani maps). Let $E$ be a Banach space, $C$ a closed convex subset of $E, U$ an open subset of $C$ and $0 \in U$. Suppose that $F: \bar{U} \rightarrow \mathcal{P}_{\mathrm{c}, \mathrm{cp}}(C)$ is an upper semicontinuous compact map. Then either

(i) F has a fixed point in $\bar{U}$, or

(ii) there are $u \in \partial U$ and $\lambda \in(0,1)$ with $u \in \lambda F(u)$.

3. Controllability for first order semilinear differential inclusions with nonlocal conditions. We give controllability results for the problem (1.1)-(1.2) when the right hand side has convex or nonconvex values. We assume first that $F: J \times E \rightarrow \mathcal{P}(E)$ is compact- and convex-valued.

Let us start by defining what we mean by a mild solution of problem $(1.1)-(1.2)$.

Definition 3.1. A function $y \in C(J, E)$ is said to be a mild solution of (1.1)-(1.2) if $y(0)+f(y)=y_{0}$ and there exists $v \in L^{1}(J, E)$ such that $v(t) \in F(t, y(t))$ a.e. on $J$, and

$$
y(t)=T(t)\left[y_{0}-f(y)\right]+\int_{0}^{t} T(t-s)[\mathcal{B} u(s)+v(s)] d s .
$$

DeFinition 3.2. The system (1.1)-(1.2) is said to be nonlocally controllable on the interval $J$ if for every $y_{0}, y_{1} \in E$ there exists a control $u \in L^{2}(J, U)$ such that there exists a mild solution $y(t)$ of (1.1)-(1.2) satisfying $y(b)+f(y)=y_{1}$.

Theorem 3.1. Let $F: J \times E \rightarrow \mathcal{P}_{\mathrm{c}, \mathrm{cp}}(E)$. Suppose that:

(3.1.1) $F$ is $L^{1}$-Carathéodory;

(3.1.2) $\mathcal{B}$ is a continuous operator from $U$ to $E$ and the linear operator $W:$ $L^{2}(J, U) \rightarrow E$, defined by

$$
W u=\int_{0}^{b} T(b-s) \mathcal{B} u(s) d s,
$$

has a bounded inverse $W^{-1}: E \rightarrow L^{2}(J, U)$ such that $\|\mathcal{B}\| \leq M_{1}$ and $\left\|W^{-1}\right\| \leq M_{2}$ for some positive constants $M_{1}, M_{2}$;

(3.1.3) $f: C(J, E) \rightarrow E$ is continuous and completely continuous (i.e. $f$ takes bounded subsets in $C(J, E)$ into relatively compact sets in $E)$ and there exists a constant $G>0$ such that $|f(y)| \leq G$ for all $y \in C(J, E)$;

(3.1.4) $A: D(A) \subset E \rightarrow E$ is the infinitesimal generator of a strongly continuous semigroup $T(t), t \geq 0$, such that for every $t>0, T(t)$ is a compact (completely continuous) operator and there exists a constant $M>0$ such that $\|T(t)\|_{B(E)} \leq M$ for all $t \geq 0$; 
(3.1.5) there exists a continuous nondecreasing function $\psi:[0, \infty) \rightarrow(0, \infty)$ and $p \in L^{1}\left(J, \mathbb{R}_{+}\right)$such that

$$
\|F(t, u)\| \leq p(t) \psi(|u|) \quad \text { for }(t, u) \in J \times E
$$

and there exists a constant $M_{*}>0$ with

$$
\frac{M_{*}}{C_{1}+\left(C_{2}+M\right) \psi\left(M_{*}\right) \int_{0}^{b} p(s) d s}>1
$$

where

$$
\begin{aligned}
& C_{1}=M\left(\left|y_{0}\right|+G\right)+b M M_{1} M_{2}\left(\left|y_{1}\right|+G+M\left|y_{0}\right|+M G\right), \\
& C_{2}=b M^{2} M_{1} M_{2}
\end{aligned}
$$

(3.1.6) given $\varepsilon>0$, for any bounded subset $D$ of $C(J, E)$ there exists a $\delta>0$ with $|(T(h)-I) f(y)|<\varepsilon$ for all $y \in D$ and $h \in[0, \delta]$.

Then the problem (1.1)-(1.2) is nonlocally controllable on J.

Proof. Using hypothesis (3.1.2), for any $y \in C(J, E)$ define the control

$$
u_{y}(t)=W^{-1}\left[y_{1}-f(y)-T(b)\left(y_{0}-f(y)\right)-\int_{0}^{b} T(b-s) v(s) d s\right](t)
$$

where

$$
v \in S_{F, y}:=\left\{v \in L^{1}(J, E): v(t) \in F(t, y(t)) \text { for a.e. } t \in J\right\} .
$$

We will show that the operator $N: C(J, E) \rightarrow \mathcal{P}(C(J, E))$ defined by

$$
\begin{aligned}
N(y):=\{h \in C(J, E): h(t)= & T(t)\left[y_{0}-f(y)\right] \\
& \left.+\int_{0}^{t} T(t-s)\left[\mathcal{B} u_{y}(s)+v(s)\right] d s: v \in S_{F, y}\right\}
\end{aligned}
$$

has a fixed point, by proving that $N$ is a completely continuous multivalued map, u.s.c. with convex values. The proof will be given in several steps.

STEP 1. $N(y)$ is convex for each $y \in C(J, E)$.

This is obvious, since $F$ has convex values.

STEP 2. $N$ maps bounded sets into bounded sets.

Indeed, it is enough to show that there exists a positive constant $\ell$ such that for each $h \in N(y)$ with $y \in B_{q}=\left\{y \in C(J, E):\|y\|=\sup _{t \in J}|y(t)|\right.$ $\leq q$ \} one has $\|h\| \leq \ell$. If $h \in N(y)$, then there exists $v \in S_{F, y}$ such that for each $t \in J$ we have 


$$
h(t)=T(t)\left[y_{0}-f(y)\right]+\int_{0}^{t} T(t-s)\left[\mathcal{B} u_{y}(s)+v(s)\right] d s .
$$

Observe that

$$
\begin{aligned}
\left|u_{y}(t)\right| & \leq M_{2}\left[\left|y_{1}\right|+G+M\left|y_{0}\right|+M G+M \int_{0}^{b}|v(s)| d s\right] \\
& \leq M_{2}\left[\left|y_{1}\right|+G+M\left|y_{0}\right|+M G+M\left\|h_{q}\right\|_{L^{1}}\right]=: H_{0}
\end{aligned}
$$

here $h_{q}$ is chosen as in Definition 2.3. Thus for each $t \in J$ we get

$$
\begin{aligned}
|h(t)| & \leq M\left|y_{0}\right|+M G+b M M_{1} H_{0}+M \int_{0}^{t}|v(s)| d s \\
& \leq M\left|y_{0}\right|+M G+b M M_{1} H_{0}+M\left\|h_{q}\right\|_{L^{1}}=: \ell .
\end{aligned}
$$

Hence for each $h \in N\left(B_{q}\right)$ we have $\|h\| \leq \ell$.

STEP 3. $N$ sends bounded sets into equicontinuous sets.

We consider $B_{q}$ as in Step 2 and let $h \in N(y)$ for $y \in B_{q}$. Let $\varepsilon>0$ and $\tau_{1}, \tau_{2} \in J$ with $\tau_{2}>\tau_{1}$. We consider two cases.

CASE 1. If $\tau_{1}>\varepsilon$ then

$$
\begin{aligned}
\left|h\left(\tau_{2}\right)-h\left(\tau_{1}\right)\right| \leq \mid & \left.\mid T\left(\tau_{2}\right)-T\left(\tau_{1}\right)\right]\left[y_{0}-f(y)\right] \mid \\
& +\int_{0}^{\tau_{1}-\varepsilon}\left|\left[T\left(\tau_{2}-s\right)-T\left(\tau_{1}-s\right)\right]\left[\mathcal{B} u_{y}(s)+v(s)\right]\right| d s \\
& +\int_{\tau_{1}-\varepsilon}^{\tau_{1}}\left|\left[T\left(\tau_{2}-s\right)-T\left(\tau_{1}-s\right)\right]\left[\mathcal{B} u_{y}(s)+v(s)\right]\right| d s \\
& +\int_{\tau_{1}}^{\tau_{2}}\left|T\left(\tau_{2}-s\right)\left[\mathcal{B} u_{y}(s)+v(s)\right]\right| d s \\
\leq & \left|\left[T\left(\tau_{2}\right)-T\left(\tau_{1}\right)\right] y_{0}\right|+M\left\|T\left(\tau_{2}-\tau_{1}+\varepsilon\right)-T(\varepsilon)\right\|_{B(E)}\left|f\left(B_{q}\right)\right| \\
& +M\left\|T\left(\tau_{2}-\tau_{1}+\varepsilon\right)-T(\varepsilon)\right\|_{B(E)} \int_{0}^{\tau_{1}-\varepsilon}\left[M_{1} H_{0}+h_{q}(s)\right] d s \\
& +2 M \int_{\tau_{1}-\varepsilon}^{\tau_{1}}\left[M_{1} H_{0}+h_{q}(s)\right] d s+M \int_{\tau_{1}}^{\tau_{2}}\left[M_{1} H_{0}+h_{q}(s)\right] d s,
\end{aligned}
$$

where we have used the semigroup identities

$$
\begin{array}{ccrl}
T\left(\tau_{2}-s\right) & =T\left(\tau_{2}-\tau_{1}+\varepsilon\right) T\left(\tau_{1}-s-\varepsilon\right), & T\left(\tau_{1}-s\right) & =T\left(\tau_{1}-s-\varepsilon\right) T(\varepsilon), \\
T\left(\tau_{2}\right) & =T\left(\tau_{2}-\tau_{1}+\varepsilon\right) T\left(\tau_{1}-\varepsilon\right), & T\left(\tau_{1}\right) & =T\left(\tau_{1}-\varepsilon\right) T(\varepsilon) .
\end{array}
$$


CASE 2. Let $\tau_{1} \leq \varepsilon$. For $\tau_{2}-\tau_{1}<\varepsilon$ we get

$$
\begin{aligned}
\left|h\left(\tau_{2}\right)-h\left(\tau_{1}\right)\right| \leq & \left|\left[T\left(\tau_{2}\right)-T\left(\tau_{1}\right)\right]\left[y_{0}-f(y)\right]\right| \\
& +\int_{0}^{\tau_{2}}\left|T\left(\tau_{2}-s\right)\left[\mathcal{B} u_{y}(s)+v(s)\right]\right| d s \\
& +\int_{0}^{\tau_{1}}\left|T\left(\tau_{1}-s\right)\left[\mathcal{B} u_{y}(s)+v(s)\right]\right| d s \\
\leq & \left|\left[T\left(\tau_{2}\right)-T\left(\tau_{1}\right)\right] y_{0}\right|+M\left|T\left(\tau_{2}-\tau_{1}\right) f(y)-f(y)\right| \\
& +M \int_{0}^{2 \varepsilon}\left[M_{1} H_{0}+h_{q}(s)\right] d s+M \int_{0}^{\varepsilon}\left[M_{1} H_{0}+h_{q}(s)\right] d s .
\end{aligned}
$$

Now equicontinuity follows since (i) $T(t), t \geq 0$, is a strongly continuous semigroup, (ii) (3.1.6) holds and (iii) $T(t)$ is compact for $t>0$ (so $T(t)$ is continuous in the uniform operator topology for $t>0$ ).

Fix $0<t \leq b$ and $0<\varepsilon<t$. For $y \in B_{q}$ and $v \in S_{F, y}$ we define

$$
\begin{aligned}
h_{\varepsilon}(t) & =T(t)\left[y_{0}-f(y)\right]+\int_{0}^{t-\varepsilon} T(t-s)\left[\mathcal{B} u_{y}(s)+v(s)\right] d s \\
& =T(t)\left[y_{0}-f(y)\right]+T(\varepsilon) \int_{0}^{t-\varepsilon} T(t-s-\varepsilon)\left[\mathcal{B} u_{y}(s)+v(s)\right] d s .
\end{aligned}
$$

Note that

$$
\left\{\int_{0}^{t-\varepsilon} T(t-s-\varepsilon)\left[\mathcal{B} u_{y}(s)+v(s)\right] d s: y \in B_{q} \text { and } v \in S_{F, y}\right\}
$$

is a bounded set since

$$
\left|\int_{0}^{t-\varepsilon} T(t-s-\varepsilon)\left[\mathcal{B} u_{y}(s)+v(s)\right] d s\right| \leq M \int_{0}^{t-\varepsilon}\left[M_{1} H_{0}+h_{q}(s)\right] d s .
$$

Now since $T(t)$ is a compact operator for $t>0$, the set $Y_{\varepsilon}(t)=\left\{h_{\varepsilon}(t): y \in\right.$ $B_{q}$ and $\left.v \in S_{F, y}\right\}$ is relatively compact in $E$ for every $0<\varepsilon<t$. Moreover for $h=h_{0}$ we have

$$
\left|h(t)-h_{\varepsilon}(t)\right| \leq M \int_{t-\varepsilon}^{t}\left[M_{1} H_{0}+h_{q}(s)\right] d s .
$$

Therefore, the set $Y(t)=\left\{h(t): y \in B_{q}\right.$ and $\left.v \in S_{F, y}\right\}$ is totally bounded. Hence $Y(t)$ is relatively compact in $E$.

As a consequence of Steps 2,3 and the Arzelà-Ascoli theorem we conclude that $N: C(J, E) \rightarrow \mathcal{P}(C(J, E))$ is completely continuous.

SteP 4. $N$ has closed graph. 
This step was proved in [3]. For completeness we include the proof. Let $y_{n} \rightarrow y_{*}, h_{n} \in N\left(y_{n}\right)$ and $h_{n} \rightarrow h_{*}$. We shall prove that $h_{*} \in N\left(y_{*}\right)$. Now $h_{n} \in N\left(y_{n}\right)$ means that there exists $v_{n} \in S_{F, y_{n}}$ such that

$$
h_{n}(t)=T(t)\left[y_{0}-f\left(y_{n}\right)\right]+\int_{0}^{t} T(t-s)\left[\mathcal{B} u_{y_{n}}(s)+v_{n}(s)\right] d s, \quad t \in J,
$$

where

$$
u_{y_{n}}(t)=W^{-1}\left[y_{1}-f\left(y_{n}\right)-T(b)\left(y_{0}-f\left(y_{n}\right)\right)-\int_{0}^{b} T(b-s) v_{n}(s) d s\right](t) .
$$

We must prove that there exists $v_{*} \in S_{F, y_{*}}$ such that

$$
h_{*}(t)=T(t)\left[y_{0}-f\left(y_{*}\right)\right]+\int_{0}^{t} T(t-s)\left[\mathcal{B} u_{y_{*}}+v_{*}(s)\right] d s, \quad t \in J,
$$

where

$$
u_{y_{*}}(t)=W^{-1}\left[y_{1}-f\left(y_{*}\right)-T(b)\left(y_{0}-f\left(y_{*}\right)\right)-\int_{0}^{b} T(b-s) v_{*}(s) d s\right](t) .
$$

Set

$$
\bar{u}_{y}(t)=W^{-1}\left[y_{1}-f(y)-T(b)\left(y_{0}-f(y)\right)\right](t) .
$$

Since $f$ and $W^{-1}$ are continuous, $\bar{u}_{y_{n}}(t) \rightarrow \bar{u}_{y^{*}}(t)$ for $t \in J$.

We have

$$
\begin{aligned}
\|\left(h_{n}-T(t)\left[y_{0}-f\left(y_{n}\right)\right]-\int_{0}^{t} T(t-s) \mathcal{B} \bar{u}_{y_{n}}(s) d s\right) \\
-\left(h_{*}-T(t)\left[y_{0}-f\left(y_{*}\right)\right]-\int_{0}^{t} T(t-s) \mathcal{B} \bar{u}_{y_{*}}(s) d s\right) \| \rightarrow 0
\end{aligned}
$$

as $n \rightarrow \infty$. Consider the operator $\Gamma: L^{1}(J, E) \rightarrow C(J, E)$ defined by

$$
(\Gamma v)(t)=\int_{0}^{t} T(t-s)\left[\mathcal{B} W^{-1}\left(\int_{0}^{b} T(b-\tau) v(\tau) d \tau\right)(s)+v(s)\right] d s .
$$

Clearly, $\Gamma$ is linear and continuous. Indeed,

$$
\|\Gamma v\| \leq b M\left(b M M_{1} M_{2}+1\right)\|v\|_{L^{1}} .
$$

It follows that $\Gamma \circ S_{F}$ has closed graph ([17]). Moreover,

$$
h_{n}(t)-T(t)\left[y_{0}-f\left(y_{n}\right)\right]-\int_{0}^{t} T(t-s) \mathcal{B} \bar{u}_{y_{n}}(s) d s \in \Gamma\left(S_{F, y_{n}}\right) .
$$


Since $y_{n} \rightarrow y_{*}$, it follows that

$$
h_{*}(t)=T(t)\left[y_{0}-f\left(y_{*}\right)\right]+\int_{0}^{t} T(t-s)\left[\mathcal{B} u_{y_{*}}(s)+v_{*}(s)\right] d s, \quad t \in J,
$$

for some $v_{*} \in S_{F, y_{*}}$.

STEP 5. There exists an open set $U \subseteq C(J, E)$ with $y \notin \lambda N y$ for $\lambda \in$ $(0,1)$ and $y \in \partial U$.

Let $\lambda \in(0,1)$ and assume $y \in \lambda N(y)$. Then there exists $v \in S_{F, y}$ such that

$$
y(t)=\lambda T(t)\left[y_{0}-f(y)\right]+\lambda \int_{0}^{t} T(t-s)\left[\mathcal{B} u_{y}(s)+v(s)\right] d s, \quad t \in J .
$$

This implies by our assumptions that for each $t \in J$ we have

$$
\begin{aligned}
|y(t)| \leq & M\left(\left|y_{0}\right|+G\right)+M \int_{0}^{t} p(s) \psi(\|y\|) d s+M \int_{0}^{t}\left|\mathcal{B} u_{y}(s)\right| d s \\
\leq & M\left(\left|y_{0}\right|+G\right)+M \int_{0}^{t} p(s) \psi(\|y\|) d s \\
& +M M_{1} M_{2} b\left(\left|y_{1}\right|+G+M\left|y_{0}\right|+M G+M \int_{0}^{b} p(s) \psi(\|y\|) d s\right) \\
\leq & C_{1}+C_{2} \psi(\|y\|) \int_{0}^{b} p(s) d s+M \psi(\|y\|) \int_{0}^{t} p(s) d s .
\end{aligned}
$$

Consequently,

$$
\frac{\|y\|}{C_{1}+\left(C_{2}+M\right) \psi(\|y\|) \int_{0}^{b} p(s) d s} \leq 1
$$

Then by (3.1.5), there exists $M_{*}$ such that $\|y\| \neq M_{*}$. Set

$$
U=\left\{y \in C(J, E):\|y\|<M_{*}\right\} .
$$

From the choice of $U$ there is no $y \in \partial U$ such that $y \in \lambda N(y)$ for some $\lambda \in(0,1)$. As a consequence of Theorem 2.2 we deduce that $N$ has a fixed point and therefore the problem (1.1)-(1.2) is nonlocally controllable.

Remark 3.1. If $\psi(u)=A u^{a}+B$ where $A \geq 0, B \geq 0,0 \leq a<1$ then clearly (3.1.5) holds since

$$
\lim _{x \rightarrow \infty} \frac{x}{C+D x^{a}}=\infty \quad \text { for any } C \geq 0, D \geq 0 .
$$

For at most linear growth of $F$ we have the following

Theorem 3.2. Assume that conditions (3.1.1)-(3.1.4) and (3.1.6) hold. In addition suppose that: 
(3.2.1) there exist $p \in L^{1}\left(J, \mathbb{R}_{+}\right)$and positive constants $A_{1}$ and $B_{1}$ such that

$$
\|F(t, u)\| \leq p(t)\left[A_{1}|u|+B_{1}\right] \quad \text { for }(t, u) \in J \times E ;
$$

$$
A_{1} C_{2} e^{A_{1} M \int_{0}^{b} p(s) d s} \int_{0}^{b} p(t) e^{-A_{1} M \int_{0}^{t} p(s) d s} d t<1, \quad C_{2}=b M^{2} M_{1} M_{2} .
$$

Then the problem (1.1)-(1.2) is nonlocally controllable on $J$.

Proof. Let $\lambda \in(0,1)$ and assume $y \in \lambda N(y)$ where $N$ is as in Theorem 3.1. For each $t \in J$ we have

$$
|y(t)| \leq C_{1}+C_{2} \int_{0}^{b} p(s)\left[A_{1}|y(s)|+B_{1}\right] d s+M \int_{0}^{t} p(s)\left[A_{1}|y(s)|+B_{1}\right] d s .
$$

Let $v(t)=\int_{0}^{t} p(s)\left[A_{1}|y(s)|+B_{1}\right] d s$. Then $v(0)=0$ and

$$
\begin{aligned}
v^{\prime}(t) & =p(t)\left[A_{1}|y(t)|+B_{1}\right] \leq p(t)\left\{A_{1}\left[C_{1}+C_{2} v(b)+M v(t)\right]+B_{1}\right\} \\
& \leq p(t) A_{1} M v(t)+p(t) A_{1} C_{2} v(b)+p(t)\left(A_{1} C_{1}+B_{1}\right) .
\end{aligned}
$$

Multiply both sides by $e^{-A_{1} M \int_{0}^{t} p(s) d s}$ to get

$$
\begin{aligned}
\left(v(t) e^{-A_{1} M \int_{0}^{t} p(s) d s}\right)^{\prime} \leq & A_{1} C_{2} v(b) p(t) e^{-A_{1} M \int_{0}^{t} p(s) d s} \\
& +p(t)\left(A_{1} C_{1}+B_{1}\right) e^{-A_{1} M \int_{0}^{t} p(s) d s} .
\end{aligned}
$$

Integrating from 0 to $b$ we get

$$
\begin{aligned}
v(b) e^{-A_{1} M \int_{0}^{b} p(s) d s} \leq & A_{1} C_{2} v(b) \int_{0}^{b} p(t) e^{-A_{1} M \int_{0}^{t} p(s) d s} d t \\
& +\left(A_{1} C_{1}+B_{1}\right) \int_{0}^{b} p(t) e^{-A_{1} M \int_{0}^{t} p(s) d s} d t
\end{aligned}
$$

or

$$
v(b) \leq \frac{\left(A_{1} C_{1}+B_{1}\right) \int_{0}^{b} p(t) e^{-A_{1} M \int_{0}^{t} p(s) d s} d t}{e^{-A_{1} M \int_{0}^{b} p(s) d s}-A_{1} C_{2} \int_{0}^{b} p(t) e^{-A_{1} M \int_{0}^{t} p(s) d s} d t}=: K_{0} .
$$

Thus $\|v\| \leq K_{0}$, so $\|y\| \leq C_{1}+\left(C_{2}+M\right) K_{0} \equiv K_{1}$. Set $M_{*}=K_{1}+1$ and now apply the nonlinear alternative as in Theorem 3.1.

Remark 3.2. Suppose $\|F(t, u)\| \leq A|u|^{a}+B$ for $A \geq 0, B \geq 0$ and $0 \leq a<1$. Now since $0 \leq a<1$ we may choose $A_{1}$ as small as we wish (in particular, so that (3.2.2) is satisfied) and $B_{1}$ large enough so that $A x^{a}+B \leq$ $A_{1} x+B_{1}$ for $x \geq 0$.

In the next theorems we weaken the boundedness assumption on the function $f$.

Theorem 3.3. Suppose (3.1.1), (3.1.2), (3.1.4) and (3.1.6) hold. In addition assume that: 
(3.3.1) $f: C(J, E) \rightarrow E$ is continuous and completely continuous and there exists a continuous nondecreasing function $\psi:[0, \infty) \rightarrow[0, \infty)$ with

$$
|f(y)| \leq \psi(\|y\|) \quad \text { for } y \in C(J, E), \quad \limsup _{q \rightarrow \infty} \frac{\psi(q)}{q}=\alpha ;
$$

(3.3.2) there exists a continuous function $p \in L^{1}[0, b]$ and a continuous nondecreasing function $g:[0, \infty) \rightarrow[0, \infty)$ such that

$$
\|F(t, y)\| \leq p(t) g(|y|), \quad t \in J, y \in E
$$

and

$$
\limsup _{q \rightarrow \infty} \frac{1}{q} g(q) \int_{0}^{b} p(s) d s=\beta,
$$

where $\alpha+\beta<1$.

Then the problem (1.1)-(1.2) is nonlocally controllable on $J$.

Proof. We show that there exists a positive integer $n_{0} \geq 1$ such that $N\left(B_{n_{0}}\right) \subset B_{n_{0}}$.

Suppose that $N\left(B_{n_{0}}\right) \nsubseteq B_{n_{0}}$ for all $n_{0} \geq 1$. Then there exist $y_{n} \in C(J, E)$ and $h_{n} \in N\left(y_{n}\right)$ such that $\left\|y_{n}\right\| \leq n$ and $\left\|h_{n}\right\|>n$. Then for every $n \geq 1$,

$$
\begin{aligned}
n<\left\|h_{n}\right\| \leq & M\left|y_{0}\right|+M \psi\left(\left\|y_{n}\right\|\right)+M \int_{0}^{t} p(s) g(n) d s \\
& +M M_{1} M_{2} b\left(\left|y_{1}\right|+\psi\left(\left\|y_{n}\right\|\right)+M\left|y_{0}\right|+M \psi\left(\left\|y_{n}\right\|\right)\right. \\
& \left.+M \int_{0}^{b} p(s) g(n) d s\right) .
\end{aligned}
$$

Divide both sides by $n$ to obtain

$$
\begin{aligned}
1< & \frac{M\left|y_{0}\right|}{n}+\frac{M \psi\left(\left\|y_{n}\right\|\right)}{n}+\frac{M}{n} \int_{0}^{t} p(s) g(n) d s \\
& +\frac{1}{n}\left[M M _ { 1 } M _ { 2 } b \left(\left|y_{1}\right|+\psi\left(\left\|y_{n}\right\|\right)+M\left|y_{0}\right|+M \psi\left(\left\|y_{n}\right\|\right)\right.\right. \\
& \left.\left.+M \int_{0}^{b} p(s) g(n) d s\right)\right] \\
\leq & \frac{M\left|y_{0}\right|}{n}+\frac{M \psi(n)}{n}+\frac{M}{n} \int_{0}^{t} p(s) g(n) d s \\
& +\frac{1}{n}\left[M M_{1} M_{2} b\left(\left|y_{1}\right|+\psi(n)+M\left|y_{0}\right|+M \psi(n)+M \int_{0}^{b} p(s) g(n) d s\right)\right] .
\end{aligned}
$$


Now take the lim sup using (3.3.1) and (3.3.2), to conclude that $1 \leq \alpha+\beta$, which is not true. Therefore there exists $n_{0} \in \mathbb{N}$ such that $N\left(B_{n_{0}}\right) \subset B_{n_{0}}$.

The proofs of the other steps are similar to those in Theorem 3.1. We omit the details.

TheOrem 3.4. Suppose (3.1.1), (3.1.2), (3.1.4) and (3.1.6) hold. In addition assume that:

(3.4.1) $f: C(J, E) \rightarrow E$ is continuous and completely continuous and there exists a continuous nondecreasing function $\psi:[0, \infty) \rightarrow[0, \infty)$ with

$$
|f(y)| \leq \psi(\|y\|) \quad \text { for } y \in C(J, E) \text {; }
$$

(3.4.2) there exist a continuous nondecreasing function $g:[0, \infty) \rightarrow(0, \infty)$ and $p \in L^{1}\left(J, \mathbb{R}_{+}\right)$such that

$$
\|F(t, u)\| \leq p(t) g(|u|) \quad \text { for }(t, u) \in J \times E
$$

and there exists a constant $M_{*}^{\prime}>0$ with

$$
\frac{M_{*}^{\prime}}{C_{1}^{\prime}+\left[M+C_{2}(1+M)\right] \psi\left(M_{*}^{\prime}\right)+\left(C_{2}+M\right) g\left(M_{*}^{\prime}\right) \int_{0}^{b} p(s) d s}>1,
$$

where

$$
C_{1}^{\prime}=M\left|y_{0}\right|+b M M_{1} M_{2}\left(\left|y_{1}\right|+M\left|y_{0}\right|\right), \quad C_{2}=b M M_{1} M_{2} .
$$

Then the problem (1.1)-(1.2) is nonlocally controllable on J.

Proof. Define $N$ as in the proof of Theorem 3.1. As there, we can prove that $N$ is completely continuous. Let $\lambda \in(0,1)$ and assume $y \in \lambda N(y)$. Then for $t \in J$ we have

$$
y(t)=\lambda T(t)\left[y_{0}-f(y)\right]+\lambda \int_{0}^{t} T(t-s)\left[\mathcal{B} u_{y}(s)+v(s)\right] d s .
$$

This implies that for each $t \in J$,

$$
\begin{aligned}
|y(t)| \leq & M\left|y_{0}\right|+M \psi(\|y\|)+M \int_{0}^{t} p(s) g(\|y\|) d s \\
& +M M_{1} M_{2} b\left(\left|y_{1}\right|+\psi(\|y\|)+M\left|y_{0}\right|+M \psi(\|y\|)\right. \\
& \left.+M \int_{0}^{b} p(s) g(\|y(s)\|) d s\right) \\
\leq & C_{1}^{\prime}+M \psi(\|y\|)+C_{2}(1+M) \psi(\|y\|) \\
& +C_{2} g(\|y\|) \int_{0}^{b} p(s) d s+M g(\|y\|) \int_{0}^{t} p(s) d s .
\end{aligned}
$$


Consequently,

$$
\frac{\|y\|}{C_{1}^{\prime}+\left(M+C_{2}(1+M)\right) \psi(\|y\|)+\left(C_{2}+M\right) g(\|y\|) \int_{0}^{b} p(s) d s} \leq 1 .
$$

Then by (3.4.2), there exists $M_{*}^{\prime}$ such that $\|y\| \neq M_{*}^{\prime}$. Set

$$
U=\left\{y \in C(J, E):\|y\|<M_{*}^{\prime}\right\} .
$$

From the choice of $U$ there is no $y \in \partial U$ such that $y=\lambda N(y)$ for some $\lambda \in(0,1)$. As a consequence of Theorem 2.2, $N$ has a fixed point $y$ in $\bar{U}$, and therefore the problem (1.1)-(1.2) is nonlocally controllable.

Next, we study the case where $F$ is not necessarily convex-valued. Our approach here is based on Theorem 2.1 combined with a selection theorem due to Bressan and Colombo [4] for lower semicontinuous multivalued operators with decomposable values.

THEOREM 3.5. Suppose that:

(3.5.1) $F: J \times E \rightarrow \mathcal{P}_{\mathrm{cp}}(E)$ and

(a) $(t, u) \mapsto F(t, u)$ is $\mathcal{L} \otimes \mathcal{B}$-measurable;

(b) $u \mapsto F(t, u)$ is lower semicontinuous for a.e. $t \in J$;

(3.5.2) for each $\varrho>0$, there exists $h_{\varrho} \in L^{1}\left(J, \mathbb{R}_{+}\right)$such that

$$
\|F(t, u)\| \leq h_{\varrho}(t) \quad \text { for a.e. } t \in J \text { and } u \in E \text { with }|u| \leq \varrho .
$$

In addition suppose (3.1.2)-(3.1.6) are satisfied. Then the initial value problem (1.1)-(1.2) has at least one solution.

Proof. Assumptions (3.5.1) and (3.5.2) imply that $F$ is lower semicontinuous. Then there exists $([4])$ a continuous map $p: C(J, E) \rightarrow L^{1}(J, E)$ such that $p(y) \in S_{F, y}$ for all $y \in C(J, E)$.

Consider the problem

$$
\begin{aligned}
y^{\prime}(t)-A y(t)-\mathcal{B} u(t) & =p(y)(t), \quad t \in J, \\
y(0)+f(y) & =y_{0} .
\end{aligned}
$$

It is obvious that if $y \in C(J, E)$ is a solution of the problem (3.1)-(3.2), then $y$ is a solution to (1.1)-(1.2).

We transform the problem (3.1)-(3.2) into a fixed point problem by considering the operator $N: C(J, E) \rightarrow C(J, E)$ defined by

$$
N(y)(t):=T(t)\left[y_{0}-f(y)\right]+\int_{0}^{t} T(t-s)\left[\mathcal{B} u_{y}(s)+p(y)(s)\right] d s .
$$

We prove that $N: C(J, E) \rightarrow C(J, E)$ is continuous.

Let $y_{n} \rightarrow y$ in $C(J, E)$. Then there is an integer $q$ such that $\left\|y_{n}\right\| \leq q$ for all $n \in \mathbb{N}$ and $\|y\| \leq q$. Then 


$$
\begin{aligned}
\mid N\left(y_{n}\right)(t)- & N(y)(t) \mid \\
\leq & M\left|f\left(y_{n}\right)-f(y)\right|+M M_{1} \int_{0}^{t}\left|u_{y_{n}}(s)-u_{y}(s)\right| d s \\
& \quad+M \int_{0}^{t}\left|p\left(y_{n}\right)(s)-p(y)(s)\right| d s \\
\leq & M\left|f\left(y_{n}\right)-f(y)\right|+M M_{1} M_{2} \int_{0}^{t}\left[(1+M)\left|f\left(y_{n}\right)-f(y)\right|\right. \\
& \left.+M \int_{0}^{b}\left|p\left(y_{n}\right)(\tau)-p(y)(\tau)\right| d \tau\right] d s+M \int_{0}^{b}\left|p\left(y_{n}\right)(s)-p(y)(s)\right| d s .
\end{aligned}
$$

Then by the dominated convergence theorem

$$
\begin{aligned}
\left\|N\left(y_{n}\right)-N(y)\right\| \leq & M\left|f\left(y_{n}\right)-f(y)\right|+b M M_{1} M_{2}(1+M)\left|f\left(y_{n}\right)-f(y)\right| \\
& +b M^{2} M_{1} M_{2}\left\|p\left(y_{n}\right)-p(y)\right\|_{L^{1}}+M\left\|p\left(y_{n}\right)-p(y)\right\|_{L^{1}} \rightarrow 0 .
\end{aligned}
$$

Thus $N$ is continuous. Also the argument in Theorem 3.1 shows that $N$ is completely continuous and that there is no $y \in \partial U$ ( $U$ as defined in Theorem 3.1) such that $y=\lambda N(y)$ for some $\lambda \in(0,1)$.

As a consequence of Theorem 2.1, $N$ has a fixed point $y$ which is a solution to problem (3.1)-(3.2), and hence the problem (1.1)-(1.2) is nonlocally controllable.

Theorem 3.6. Assume that (3.1.2), (3.1.4), (3.1.6), (3.4.1), (3.4.2), (3.5.1) and (3.5.2) are satisfied. Then the problem (1.1)-(1.2) is nonlocally controllable on $J$.

4. A special case. In this section we consider a special case of the nonlocal condition, i.e. we consider the following problem:

$$
\begin{gathered}
y^{\prime}(t) \in A y(t)+F(t, y(t))+\mathcal{B} u(t), \quad t \in J:=[0, b], \\
y(0)+\sum_{k=1}^{p} c_{k} y\left(t_{k}\right)=y_{0},
\end{gathered}
$$

where $A, F, y_{0}, \mathcal{B}, u$ are as in (1.1)-(1.2) and $0 \leq t_{1}<\cdots<t_{p} \leq b, p \in \mathbb{N}$, $c_{k} \neq 0, k=1, \ldots, p$.

As remarked by Byszewski [6] if $c_{k} \neq 0, k=1, \ldots, p$, the results can be applied to kinematics to determine the evolution $t \mapsto y(t)$ of the location of a physical object for which we do not know the positions $y(0), y\left(t_{1}\right), \ldots, y\left(t_{p}\right)$, but instead we know that the nonlocal condition (4.2) holds. Consequently, to describe some physical phenomena, the nonlocal condition can be more useful than the standard initial condition $y(0)=y_{0}$. From (4.2) it is clear that when $c_{k}=0, k=1, \ldots, p$, we have the classical initial condition. 
A controllability result for the problem (4.1)-(4.2) was given in [3]. However, the result in [3] is incorrect since the constant $\widehat{M}$ on page 74 depends on the solution. Here we prove controllability results for (4.1)-(4.2) using Theorem 2.2.

In the following we assume that the following condition is satisfied:

(B1) The inverse operator

$$
\Theta:=\left(I+\sum_{k=1}^{p} c_{k} T\left(t_{k}\right)\right)^{-1}
$$

exists and $\Theta \in B(E)$.

Notice that $\Theta$ exists if $M \sum_{k=1}^{p}\left|c_{k}\right|<1$.

Definition 4.1. A function $y \in C(J, E)$ is said to be a mild solution of (4.1)-(4.2) if $y(0)+\sum_{k=1}^{p} c_{k} y\left(t_{k}\right)=y_{0}$ and there exists $v \in L^{1}(J, E)$ such that $v(t) \in F(t, y(t))$ a.e. on $J$, and

$$
\begin{aligned}
y(t)= & T(t) \Theta y_{0}-\sum_{k=1}^{p} c_{k} T(t) \Theta \int_{0}^{t_{k}} T\left(t_{k}-s\right) v(s) d s \\
& +\int_{0}^{t} T(t-s)[\mathcal{B} u(s)+v(s)] d s .
\end{aligned}
$$

Definition 4.2. The system (4.1)-(4.2) is said to be nonlocally controllable on the interval $J$ if for every $y_{0}, y_{1} \in E$ there exists a control $u \in L^{2}(J, U)$ such that there exists a mild solution $y(t)$ of $(4.1)-(4.2)$ satisfying $y(b)+\sum_{k=1}^{p} c_{k} y\left(t_{k}\right)=y_{1}$.

Theorem 4.1. Let $F: J \times E \rightarrow \mathcal{P}_{\mathrm{c}, \mathrm{cp}}(E)$. Assume that (B1), (3.1.1), (3.1.2) and (3.1.4) hold. In addition suppose that:

(4.1.1) there exists a continuous nondecreasing function $\psi:[0, \infty) \rightarrow(0, \infty)$ and $p \in L^{1}\left(J, \mathbb{R}_{+}\right)$such that

$$
\|F(t, u)\| \leq p(t) \psi(|u|) \quad \text { for }(t, u) \in J \times E
$$

and there exists a constant $M_{* *}>0$ with

$$
\frac{\left(1-K_{1} \sum_{k=1}^{p}\left|c_{k}\right|\right) M_{* *}}{K_{2}+K_{3} \psi\left(M_{* *}\right) \int_{0}^{t_{k}} p(t) d t+M\left(1+K_{1}\right) \psi\left(M_{* *}\right) \int_{0}^{b} p(s) d s}>1,
$$

where

$$
\begin{aligned}
& K_{1}=b M M_{1} M_{2}, \quad K_{2}=M\|\Theta\|_{B(E)}\left|y_{0}\right|+K_{2}\left(\left|y_{1}\right|+M\|\Theta\|_{B(E)}\left|y_{0}\right|\right), \\
& K_{3}=\left(1+K_{1}\right) M^{2}\|\Theta\|_{B(E)} \sum_{k=1}^{p}\left|c_{k}\right|
\end{aligned}
$$


and

$$
K_{1} \sum_{k=1}^{p}\left|c_{k}\right|<1 ;
$$

(4.1.2) given $\varepsilon>0$, there exists a $\delta>0$ with $\|T(h)-I\|_{B(E)}<\varepsilon$ for all $h \in[0, \delta]$.

Then the nonlocal problem (4.1)-(4.2) is nonlocally controllable on J.

Proof. Using hypothesis (3.1.2), for $y \in C(J, E)$ define the control

$$
\begin{aligned}
u_{y}(t)= & W^{-1}\left[y_{1}-\sum_{k=1}^{p} c_{k} y\left(t_{k}\right)-T(b) \Theta y_{0}+\sum_{k=1}^{p} c_{k} T(b) \Theta \int_{0}^{t_{k}} T\left(t_{k}-s\right) v(s) d s\right. \\
& \left.-\int_{0}^{b} T(b-s) v(s) d s\right](t),
\end{aligned}
$$

where $v \in S_{F, y}$. We will show that the operator $N: C(J, E) \rightarrow \mathcal{P}(C(J, E))$ defined by

$$
\begin{aligned}
N(y):=\{h \in C(J, E): h(t)= & T(t) \Theta y_{0}-\sum_{k=1}^{p} c_{k} T(t) \Theta \int_{0}^{t_{k}} T\left(t_{k}-s\right) v(s) d s \\
& \left.+\int_{0}^{t} T(t-s)\left[\mathcal{B} u_{y}(s)+v(s)\right] d s: v \in S_{F, y}\right\}
\end{aligned}
$$

has a fixed point. This fixed point is then a solution of the system (4.1)-(4.2). Note that $y_{1}-\sum_{k=1}^{p} c_{k} y\left(t_{k}\right) \in(N y)(b)$.

We shall show that $N$ is completely continuous with bounded, closed, convex values and it is upper semicontinuous. The proof mimicks the proof of Theorem 3.1.

STEP 1. $N(y)$ is convex for each $y \in C(J, E)$.

This is obvious, since $F$ has convex values.

STEP 2. $N$ maps bounded sets into bounded sets.

Indeed, if $h \in N(y)$ and $y \in B_{q}$, then there exists $v \in S_{F, y}$ such that for each $t \in J$,

$$
\begin{aligned}
h(t)= & T(t) \Theta y_{0}-\sum_{k=1}^{p} c_{k} T(t) \Theta \int_{0}^{t_{k}} T\left(t_{k}-s\right) v(s) d s \\
& +\int_{0}^{t} T(t-s)\left[\mathcal{B} u_{y}(s)+v(s)\right] d s .
\end{aligned}
$$

Observe that 


$$
\begin{aligned}
\left|u_{y}(t)\right| \leq & M_{2}\left[\left|y_{1}\right|+\sum_{k=1}^{p}\left|c_{k}\right|\left|y\left(t_{k}\right)\right|+M\|\Theta\|_{B(E)}\left|y_{0}\right|\right. \\
& \left.+\sum_{k=1}^{p}\left|c_{k}\right| M^{2}\|\Theta\|_{B(E)} \int_{0}^{t_{k}} h_{q}(s) d s+M \int_{0}^{b} h_{q}(s) d s\right] \\
\leq & M_{2}\left[\left|y_{1}\right|+\sum_{k=1}^{p}\left|c_{k}\right| q+M\|\Theta\|_{B(E)}\left|y_{0}\right|\right. \\
& \left.+\sum_{k=1}^{p}\left|c_{k}\right| M^{2}\|\Theta\|_{B(E)} \int_{0}^{t_{k}} h_{q}(s) d s+M\left\|h_{q}\right\|_{L^{1}}\right]:=H_{1},
\end{aligned}
$$

with $h_{q}$ as in Definition 2.3. Thus for each $t \in J$,

$$
\begin{aligned}
|h(t)| \leq & M\|\Theta\|_{B(E)}\left|y_{0}\right|+M^{2}\|\Theta\|_{B(E)} \sum_{k=1}^{p}\left|c_{k}\right| \int_{0}^{t_{k}} h_{q}(s) d s \\
& +b M M_{1} H_{1}+M\left\|h_{q}\right\|_{L^{1}} .
\end{aligned}
$$

STEP 3. $N$ sends bounded sets into equicontinuous sets.

Let $h \in N(y)$ for $y \in B_{q}$. Let $\varepsilon>0$ and $\tau_{1}, \tau_{2} \in J$ with $\tau_{2}>\tau_{1}$.

CASE 1. If $\tau_{1}>\varepsilon$ then

$$
\begin{aligned}
& \left|h\left(\tau_{2}\right)-h\left(\tau_{1}\right)\right| \leq\left|\left[T\left(\tau_{2}\right)-T\left(\tau_{1}\right)\right] \Theta y_{0}\right| \\
& +M\|\Theta\|_{B(E)}\left\|T\left(\tau_{2}\right)-T\left(\tau_{1}\right)\right\|_{B(E)} \sum_{k=1}^{p}\left|c_{k}\right| \int_{0}^{t_{k}}|v(s)| d s \\
& +\int_{0}^{\tau_{1}-\varepsilon}\left|\left[T\left(\tau_{2}-s\right)-T\left(\tau_{1}-s\right)\right]\left[\mathcal{B} u_{y}(s)+v(s)\right]\right| d s \\
& +\int_{\tau_{1}-\varepsilon}^{\tau_{1}}\left|\left[T\left(\tau_{2}-s\right)-T\left(\tau_{1}-s\right)\right]\left[\mathcal{B} u_{y}(s)+v(s)\right]\right| d s \\
& +\int_{\tau_{1}}^{\tau_{2}}\left|T\left(\tau_{2}-s\right)\left[\mathcal{B} u_{y}(s)+v(s)\right]\right| d s \\
& \leq\left|\left[T\left(\tau_{2}\right)-T\left(\tau_{1}\right)\right] \Theta y_{0}\right| \\
& +M\|\Theta\|_{B(E)}\left\|T\left(\tau_{2}\right)-T\left(\tau_{1}\right)\right\|_{B(E)} \sum_{\substack{k=1 \\
\tau_{1}-\varepsilon}}^{p}\left|c_{k}\right| \int_{0}^{t_{k}} h_{q}(s) d s \\
& +M\left\|T\left(\tau_{2}-\tau_{1}+\varepsilon\right)-T(\varepsilon)\right\|_{B(E)} \int_{0}^{\tau_{1}-\varepsilon}\left[M_{1} H_{1}+h_{q}(s)\right] d s \\
& +2 M \int_{\tau_{1}-\varepsilon}^{\tau_{1}}\left[M_{1} H_{1}+h_{q}(s)\right] d s+M \int_{\tau_{1}}^{\tau_{2}}\left[M_{1} H_{1}+h_{q}(s)\right] d s .
\end{aligned}
$$


CASE 2. Let $\tau_{1} \leq \varepsilon$. For $\tau_{2}-\tau_{1}<\varepsilon$ we get

$$
\begin{aligned}
\left|h\left(\tau_{2}\right)-h\left(\tau_{1}\right)\right| \leq & \left|\left[T\left(\tau_{2}\right)-T\left(\tau_{1}\right)\right] \Theta y_{0}\right| \\
& +M\|\Theta\|_{B(E)}\left\|T\left(\tau_{2}\right)-T\left(\tau_{1}\right)\right\|_{B(E)} \sum_{k=1}^{p}\left|c_{k}\right| \int_{0}^{t_{k}} h_{q}(s) d s \\
& +\int_{0}^{\tau_{2}}\left\|T\left(\tau_{2}-s\right)\right\|_{B(E)}\left[M_{1} H_{1}+h_{q}(s)\right] d s \\
& +\int_{0}^{\tau_{1}}\left\|T\left(\tau_{2}-s\right)\right\|_{B(E)}\left[M_{1} H_{1}+h_{q}(s)\right] d s \\
\leq & \left|\left[T\left(\tau_{2}\right)-T\left(\tau_{1}\right)\right] \Theta y_{0}\right| \\
& +M^{2}\|\Theta\|_{B(E)}\left\|T\left(\tau_{2}-\tau_{1}\right)-I\right\|_{B(E)} \sum_{k=1}^{p}\left|c_{k}\right| \int_{0}^{t_{k}} h_{q}(s) d s \\
& +\int_{0}^{2 \varepsilon}\left[M_{1} H_{1}+h_{q}(s)\right] d s+M \int_{0}^{\varepsilon}\left[M_{1} H_{1}+h_{q}(s)\right] d s .
\end{aligned}
$$

Now equicontinuity follows as in Theorem 3.1, with (4.1.2) replacing (3.1.6).

Fix $0<t \leq b$ and $0<\varepsilon<t$. For $y \in B_{q}$ and $v \in S_{F, y}$ we define

$$
\begin{aligned}
h_{\varepsilon}(t)= & T(t) \Theta y_{0}-\sum_{k=1}^{p} c_{k} T(t) \Theta \int_{0}^{t_{k}} T\left(t_{k}-s\right) v(s) d s \\
& +\int_{0}^{t-\varepsilon} T(t-s)\left[\mathcal{B} u_{y}(s)+v(s)\right] d s \\
= & T(t) \Theta y_{0}-\sum_{k=1}^{p} c_{k} T(t) \Theta \int_{0}^{t_{k}} T\left(t_{k}-s\right) v(s) d s \\
& +T(\varepsilon) \int_{0}^{t-\varepsilon} T(t-s-\varepsilon)\left[\mathcal{B} u_{y}(s)+v(s)\right] d s .
\end{aligned}
$$

Note that

$$
\left|\Theta \int_{0}^{t_{k}} T\left(t_{k}-s\right) v(s) d s\right| \leq M\|\Theta\|_{B(E)} \int_{0}^{t_{k}} h_{q}(s) d s .
$$

Also

$$
\left\{\int_{0}^{t-\varepsilon} T(t-s-\varepsilon)\left[\mathcal{B} u_{y}(s)+v(s)\right] d s: y \in B_{q} \text { and } v \in S_{F, y}\right\}
$$

is a bounded set since

$$
\left|\int_{0}^{t-\varepsilon} T(t-s-\varepsilon)\left[\mathcal{B} u_{y}(s)+v(s)\right] d s\right| \leq M \int_{0}^{t-\varepsilon}\left[M_{1} H_{1}+h_{q}(s)\right] d s .
$$


Now since $T(t)$ is compact for $t>0$, the set $Y_{\varepsilon}(t)=\left\{h_{\varepsilon}(t): y \in B_{q}\right.$ and $\left.v \in S_{F, y}\right\}$ is relatively compact in $E$ for every $0<\varepsilon<t$. Moreover for $h=h_{0}$,

$$
\left|h(t)-h_{\varepsilon}(t)\right| \leq M \int_{t-\varepsilon}^{t}\left[M_{1} H_{1}+h_{q}(s)\right] d s .
$$

Therefore, $Y(t)=\left\{h(t): y \in B_{q}\right.$ and $\left.v \in S_{F, y}\right\}$ is totally bounded and hence $Y(t)$ is relatively compact in $E$.

As a consequence of Steps 2,3 and the Arzelà-Ascoli theorem we conclude that $N: C(J, E) \rightarrow \mathcal{P}(C(J, E))$ is completely continuous.

STEP 4. $N$ has closed graph.

This step was proved in [3]. For completeness we include the proof. Let $y_{n} \rightarrow y_{*}, h_{n} \in N\left(y_{n}\right)$ and $h_{n} \rightarrow h_{*}$. There exists $v_{n} \in S_{F, y_{n}}$ such that

$$
\begin{aligned}
h_{n}(t)= & T(t) \Theta y_{0}-\sum_{k=1}^{p} c_{k} T(t) \Theta \int_{0}^{t_{k}} T\left(t_{k}-s\right) v_{n}(s) d s \\
& +\int_{0}^{t} T(t-s)\left[\mathcal{B} u_{y_{n}}(s)+v_{n}(s)\right] d s, \quad t \in J,
\end{aligned}
$$

where $u_{y}(t)$ is as defined at the beginning of the proof. We must prove that there exists $v_{*} \in S_{F, y_{*}}$ such that

$$
\begin{aligned}
h_{*}(t)= & T(t) \Theta y_{0}-\sum_{k=1}^{p} c_{k} T(t) \Theta \int_{0}^{t_{k}} T\left(t_{k}-s\right) v_{*}(s) d s \\
& +\int_{0}^{t} T(t-s)\left[\mathcal{B} u_{y_{*}}(s)+v_{*}(s)\right] d s, \quad t \in J .
\end{aligned}
$$

Set

$$
\bar{u}_{y}=W^{-1}\left[y_{1}-\sum_{k=1}^{p} c_{k} y\left(t_{k}\right)-T(b) \Theta y_{0}+\sum_{k=1}^{p} c_{k} T(b) \Theta \int_{0}^{t_{k}} T\left(t_{k}-s\right) v(s) d s\right] .
$$

Since $f$ and $W^{-1}$ are continuous, $\bar{u}_{y_{n}}(t) \rightarrow \bar{u}_{y^{*}}(t)$ for $t \in J$.

We have

$$
\begin{aligned}
\|\left(h_{n}-T(t) \Theta y_{0}-\int_{0}^{t} T(t-s) \mathcal{B} \bar{u}_{y_{n}}(s) d s\right) \\
\quad-\left(h_{*}-T(t) \Theta y_{0}-\int_{0}^{t} T(t-s) \mathcal{B} \bar{u}_{y_{*}}(s) d s\right) \| \rightarrow 0 \quad \text { as } n \rightarrow \infty .
\end{aligned}
$$

Consider the operator $\Gamma: L^{1}(J, E) \rightarrow C(J, E)$ defined by 


$$
\begin{aligned}
(\Gamma v)(t)= & \int_{0}^{t} T(t-s) v(s) d s-\sum_{k=1}^{p} c_{k} T(b) \Theta \int_{0}^{t_{k}} T\left(t_{k}-s\right) v(s) d s \\
& +\int_{0}^{t} T(t-s) \mathcal{B} W^{-1}\left(\int_{0}^{b} T(b-\tau) v(\tau) d \tau\right)(s) d s .
\end{aligned}
$$

Clearly, $\Gamma$ is linear and continuous. Indeed,

$$
\|\Gamma v\| \leq M\left(1+M\|\Theta\|_{B(E)} \sum_{k=1}^{p}\left|c_{k}\right|+b M M_{1} M_{2}\right)\|v\|_{L^{1}} .
$$

It follows that $\Gamma \circ S_{F}$ has closed graph ([17]). Moreover,

$$
h_{n}(t)-T(t) \Theta y_{0}-\int_{0}^{t} T(t-s) \mathcal{B} \bar{u}_{y_{n}}(s) \in \Gamma\left(S_{F, y_{n}}\right) .
$$

Since $y_{n} \rightarrow y_{*}$, it follows that

for some $v_{*} \in S_{F, y_{*}}$.

$$
\begin{aligned}
h_{*}(t)= & T(t) \Theta y_{0}-\sum_{k=1}^{p} c_{k} T(t) \Theta \int_{0}^{t_{k}} T\left(t_{k}-s\right) v_{*}(s) d s \\
& +\int_{0}^{t} T(t-s)\left[\mathcal{B} u_{y_{*}}(s)+v_{*}(s)\right] d s, \quad t \in J,
\end{aligned}
$$

STEP 5. There exists an open set $U \subseteq C(J, E)$ with $y \notin \lambda N y$ for $\lambda \in$ $(0,1)$ and $y \in \partial U$.

Let $\lambda \in(0,1)$ and assume $y \in \lambda N(y)$. Then for $t \in J$ we have

$$
\begin{aligned}
y(t)= & \lambda T(t) \Theta y_{0}-\lambda \sum_{k=1}^{p} c_{k} T(t) \Theta \int_{0}^{t_{k}} T\left(t_{k}-s\right) v(s) d s \\
& +\lambda \int_{0}^{t} T(t-s)\left[\mathcal{B} u_{y}(s)+v(s)\right] d s .
\end{aligned}
$$

This implies that for each $t \in J$,

$$
\begin{aligned}
|y(t)| \leq & M\|\Theta\|_{B(E)}\left|y_{0}\right|+M^{2}\|\Theta\|_{B(E)} \sum_{k=1}^{p}\left|c_{k}\right| \int_{0}^{t_{k}} p(s) \psi(\|y\|) d s \\
& +b M M_{1} M_{2}\left[\left|y_{1}\right|+\sum_{k=1}^{p}\left|c_{k}\right|\|y\|+M\|\Theta\|_{B(E)}\left|y_{0}\right|\right. \\
& \left.+\sum_{k=1}^{p}\left|c_{k}\right| M^{2}\|\Theta\|_{B(E)} \int_{0}^{t_{k}} p(s) \psi(\|y\|) d s+M \int_{0}^{b} p(s) \psi(\|y\|) d s\right] \\
& +M \int_{0}^{t} p(s) \psi(\|y\|) d s
\end{aligned}
$$




$$
\begin{aligned}
\leq & K_{2}+\left(1+K_{1}\right) M^{2}\|\Theta\|_{B(E)} \sum_{k=1}^{p}\left|c_{k}\right| \psi(\|y\|) \int_{0}^{t_{k}} p(s) d s \\
& +K_{1} \sum_{k=1}^{p}\left|c_{k}\right|\|y\|+M\left(1+K_{1}\right) \psi(\|y\|) \int_{0}^{b} p(s) d s .
\end{aligned}
$$

Consequently,

$$
\frac{\left(1-K_{1} \sum_{k=1}^{p}\left|c_{k}\right|\right)\|y\|}{K_{2}+K_{3} \psi(\|y\|) \int_{0}^{t_{k}} p(t) d t+M\left(1+K_{1}\right) \psi(\|y\|) \int_{0}^{b} p(s) d s} \leq 1 .
$$

Then by (4.1.1), there exists $M_{* *}$ such that $\|y\| \neq M_{* *}$. Set

$$
U=\left\{y \in C(J, E):\|y\|<M_{* *}\right\}
$$

and apply Theorem 2.2 to conclude the proof.

If we have at most linear growth then we have the following

THEOREM 4.2. Assume that (3.1.1), (3.1.2), (3.1.4), (B1) and (4.1.2) hold. In addition suppose that:

(4.2.1) there exists $p \in L^{1}\left(J, \mathbb{R}_{+}\right)$and positive constants $A_{1}$ and $B_{1}$ such that

$$
\|F(t, u)\| \leq p(t)\left[A_{1}|u|+B_{1}\right] \quad \text { for }(t, u) \in J \times E
$$

(4.2.2) $A_{1} K_{2} e^{-A_{1} M \int_{0}^{b} p(s) d s} \int_{0}^{b} p(t) e^{-A_{1} M \int_{0}^{t} p(s) d s} d t<1$

$$
K_{1}=M\|\Theta\|_{B(E)}\left|y_{0}\right|, \quad K_{2}=M^{2}\|\Theta\|_{B(E)} \sum_{k=1}^{p}\left|c_{k}\right| .
$$

Then the problem (4.1)-(4.2) is nonlocally controllable on J.

Proof. Let $\lambda \in(0,1)$ and assume $y=\lambda N(y)$ where $N$ is as in Theorem 4.1. For each $t \in J$ we have

$$
|y(t)| \leq K_{1}+K_{2} \sum_{k=1}^{p}\left|c_{k}\right| \int_{0}^{t_{k}} p(s)\left[A_{1}|y(s)|+B_{1}\right] d s+M \int_{0}^{t} p(s)\left[A_{1}|y(s)|+B_{1}\right] d s .
$$

Let $v(t)=\int_{0}^{t} p(s)\left[A_{1}|y(s)|+B_{1}\right] d s$. Then $v(0)=0$ and

$$
\begin{aligned}
v^{\prime}(t) & \leq p(t) A_{1} K_{2} \sum_{k=1}^{p}\left|c_{k}\right| v\left(t_{k}\right)+p(t) A_{1} M v(t)+p(t)\left[A_{1} K_{1}+B_{1}\right] \\
& \leq p(t) A_{1} K_{2} \sum_{k=1}^{p}\left|c_{k}\right| v(b)+p(t) A_{1} M v(t)+p(t)\left[A_{1} K_{1}+B_{1}\right]
\end{aligned}
$$


Multiply both sides by $e^{-A_{1} M \int_{0}^{t} p(s) d s}$ to get

$$
\begin{aligned}
\left(v(t) e^{-A_{1} M \int_{0}^{t} p(s) d s}\right)^{\prime} \leq & p(t) A_{1} K_{2} \sum_{k=1}^{p}\left|c_{k}\right| v(b) e^{-A_{1} M \int_{0}^{t} p(s) d s} \\
& +p(t)\left[A_{1} K_{1}+B_{1}\right] e^{-A_{1} M \int_{0}^{t} p(s) d s} .
\end{aligned}
$$

Integrate from 0 to $b$ to obtain

$$
\begin{aligned}
v(b) e^{-A_{1} M \int_{0}^{b} p(s) d s} \leq & A_{1} K_{2} v(b) \sum_{k=1}^{p}\left|c_{k}\right| \int_{0}^{b} p(t) e^{-A_{1} M \int_{0}^{t} p(s) d s} d t \\
& +\left[A_{1} K_{1}+B_{1}\right] \int_{0}^{b} p(t) e^{-A_{1} M \int_{0}^{t} p(s) d s} d t
\end{aligned}
$$

or

$$
v(b) \leq \frac{\left[A_{1} K_{1}+B_{1}\right] \int_{0}^{b} p(t) e^{-A_{1} M \int_{0}^{t} p(s) d s}}{e^{-A_{1} M \int_{0}^{b} p(s) d s}-A_{1} K_{2} \sum_{k=1}^{p}\left|c_{k}\right| \int_{0}^{b} p(t) e^{-A_{1} M \int_{0}^{t} p(s) d s}}=: K^{\prime} .
$$

Thus $\|v\| \leq K^{\prime}$, so $\|y\| \leq K_{1}+\left(K_{2} \sum_{k=1}^{p}\left|c_{k}\right|+M\right) K^{\prime} \equiv K_{1}^{\prime}$. Set $M_{*}=K_{1}^{\prime}+1$ and apply the nonlinear alternative as in Theorem 4.1.

For the lower semicontinuous case we state without proof the following results.

Theorem 4.3. Assume that (3.1.2), (3.1.4), (3.5.1), (3.5.2), (B1), (4.1.1) and (4.1.2) are satisfied. Then the problem (4.1)-(4.2) is nonlocally controllable on $\mathrm{J}$.

Theorem 4.4. Assume that (3.1.2), (3.1.4), (3.5.1), (3.5.2), (B1), (4.1.2), (4.2.1) and (4.2.2) are satisfied. Then the nonlocal problem (4.1)-(4.2) is nonlocally controllable on $\mathrm{J}$.

\section{Controllability for semilinear evolution inclusions with non-} local conditions and nondense domain. In Theorem 3.1 the operator $A$ was densely defined. However, as indicated in [7], we sometimes need to deal with nondensely defined operators. For example, when we look at a one-dimensional heat equation with Dirichlet conditions on $[0,1]$ and consider $A=\partial^{2} / \partial x^{2}$ in $C([0,1], \mathbb{R})$ in order to measure the solutions in the sup-norm, then the domain

$$
D(A)=\left\{\phi \in C^{2}([0,1], \mathbb{R}): \phi(0)=\phi(1)=0\right\}
$$

is not dense in $C([0,1], \mathbb{R})$ with the sup-norm. See [7] for more examples and remarks concerning nondensely defined operators. We can extend the results for problem (1.1)-(1.2) to the case where $A$ is nondensely defined. The basic tool for this study is the theory of integrated semigroups. 
Definition 5.1 ([1]). Let $E$ be a Banach space. An integrated semigroup is a family $(S(t))_{t \geq 0}$ of bounded linear operators $S(t)$ on $E$ with the following properties:

(i) $S(0)=0$;

(ii) $t \mapsto S(t)$ is strongly continuous;

(iii) $S(s) S(t)=\int_{0}^{s}(S(t+r)-S(r)) d r$ for all $t, s \geq 0$.

If $A$ is the generator of an integrated semigroup $(S(t))_{t \geq 0}$ which is locally Lipschitz, then from [1], $S(\cdot) x$ is continuously differentiable if and only if $x \in \overline{D(A)}$. In particular $S^{\prime}(t) x:=(d / d t) S(t) x$ defines a bounded operator on the set $E_{1}:=\{x \in E: t \mapsto S(t) x$ is continuously differentiable on $[0, \infty)\}$ and $\left(S^{\prime}(t)\right)_{t \geq 0}$ is a $C_{0}$ semigroup on $\overline{D(A)}$. Here and hereafter, we assume that $A$ satisfies the Hille-Yosida condition.

Let $(S(t))_{t \geq 0}$ be the integrated semigroup generated by $A$. We note that, since $A$ satisfies the Hille-Yosida condition, $\left\|S^{\prime}(t)\right\|_{B(E)} \leq M e^{\omega t}, t \geq 0$, where $M$ and $\omega$ are from the Hille-Yosida condition (see [16]).

We give some results on the existence of solutions of the problem

$$
\begin{aligned}
& y^{\prime}(t)=A y(t)+f(t), \quad t \geq 0, \\
& y(0)=y_{0} \in E,
\end{aligned}
$$

where $A$ satisfies the Hille-Yosida condition, without being densely defined.

THEOREM 5.1 ([16]). Let $f: J \rightarrow E$ be a continuous function. Then for $y_{0} \in \overline{D(A)}$, there exists a unique continuous function $y: J \rightarrow E$ such that
(i) $\int_{0}^{t} y(s) d s \in D(A)$ for $t \in J$,
(ii) $y(t)=y_{0}+A \int_{0}^{t} y(s) d s+\int_{0}^{t} f(s) d s, \quad t \in J$,
(iii) $|y(t)| \leq M e^{\omega t}\left(\left|y_{0}\right|+\int_{0}^{t} e^{-\omega s}|f(s)| d s\right), \quad t \in J$.

Moreover, $y$ satisfies the following variation of constants formula:

$$
y(t)=S^{\prime}(t) y_{0}+\frac{d}{d t} \int_{0}^{t} S(t-s) f(s) d s, \quad t \geq 0 .
$$

Let $B_{\lambda}=\lambda R(\lambda, A):=\lambda(\lambda I-A)^{-1}$. Then ([16]) for all $x \in \overline{D(A)}$ we have $B_{\lambda} x \rightarrow x$ as $\lambda \rightarrow \infty$. Also from the Hille-Yosida condition (with $n=1$ ) it is easy to see that $\lim _{\lambda \rightarrow \infty}\left|B_{\lambda} x\right| \leq M|x|$, since

$$
\left\|B_{\lambda}\right\|=\left\|\lambda(\lambda I-A)^{-1}\right\| \leq \frac{M \lambda}{\lambda-\omega} .
$$


Thus $\lim _{\lambda \rightarrow \infty}\left\|B_{\lambda}\right\| \leq M$. Also if $y$ satisfies (5.3), then

$$
y(t)=S^{\prime}(t) y_{0}+\lim _{\lambda \rightarrow \infty} \int_{0}^{t} S^{\prime}(t-s) B_{\lambda} f(s) d s, \quad t \geq 0 .
$$

We are now in a position to define what we mean by an integral solution of the problem (1.1)-(1.2).

Definition 5.2. We say that $y: J \rightarrow E$ is an integral solution of (1.1)(1.2) if

(i) $y \in C(J, E)$,

(ii) $\int_{0}^{t} y(s) d s \in D(A)$ for $t \in J$,

(iii) there exists a function $v \in L^{1}(J, E)$ such that $v(t) \in F(t, y(t))$ a.e. in $J$ and

$$
y(t)=S^{\prime}(t)\left[y_{0}-f(y)\right]+\frac{d}{d t} \int_{0}^{t} S(t-s)[\mathcal{B} u(s)+v(s)] d s .
$$

From (ii) we know that $y(t) \in \overline{D(A)}$ for all $t \geq 0$. Also from (iii) we deduce that $y_{0}-f(y) \in \overline{D(A)}$. Hence, if $y_{0} \in \overline{D(A)}$ then $f(y) \in \overline{D(A)}$.

Theorem 5.2. Assume that (3.1.1), (3.1.3) and (3.1.4) hold and in addition:

(5.2.1) A satisfies the Hille-Yosida condition;

(5.2.2) $S^{\prime}(t)$ is compact in $\overline{D(A)}$ whenever $t>0$;

(5.2.3) $y_{0} \in \overline{D(A)}$;

(5.2.4) $\mathcal{B}$ is a continuous operator from $U$ to $E$ and the linear operator $W$ : $L^{2}(J, U) \rightarrow E$, defined by

$$
W u=\lim _{\lambda \rightarrow \infty} \int_{0}^{b} S^{\prime}(b-s) B_{\lambda} \mathcal{B} u(s) d s,
$$

has a bounded inverse $W^{-1}: E \rightarrow L^{2}(J, U)$ such that $\|\mathcal{B}\| \leq M_{1}$ and $\left\|W^{-1}\right\| \leq M_{2}$ for some positive constants $M_{1}, M_{2}$;

(5.2.5) there exists a continuous nondecreasing function $\psi:[0, \infty) \rightarrow(0, \infty)$ and $p \in L^{1}\left(J, \mathbb{R}_{+}\right)$such that

$$
\|F(t, u)\| \leq p(t) \psi(|u|) \quad \text { for }(t, u) \in J \times E
$$

and there exists a constant $M_{*}>0$ with

$$
\frac{M_{*}}{Z_{2}+M^{*}\left(1+Z_{1}\right) \psi\left(M_{*}\right) \int_{0}^{b} p(s) d s}>1,
$$

where

$Z_{1}=b M^{*} M_{1} M_{2}, \quad Z_{2}=M^{*}\left[\left|y_{0}\right|+G\right]+Z_{1}\left[\left|y_{1}\right|+G+M^{*}\left(\left|y_{0}\right|+G\right)\right]$ and $M^{*}=\max \left\{e^{\omega b}, 1\right\}$; 
(5.2.6) given $\varepsilon>0$, for any bounded subset $D$ of $C(J, E)$ there exists a $\delta>0$ with $\left|\left[S^{\prime}(h)-I\right] f(y)\right|<\varepsilon$ for all $y \in D$ and $h \in[0, \delta]$.

Then the problem (1.1)-(1.2) has at least one integral solution on $J$.

Proof. Using hypothesis (5.2.4), for any $y \in C(J, E)$ define the control

$$
u_{y}(t)=W^{-1}\left[y_{1}-f(y)-S^{\prime}(b)\left(y_{0}-f(y)\right)-\lim _{\lambda \rightarrow \infty} \int_{0}^{b} S^{\prime}(b-s) B_{\lambda} v(s) d s\right](t),
$$

where $v \in S_{F, y}$. We will show that the operator $N: C(J, E) \rightarrow \mathcal{P}(C(J, E))$ defined by

$$
\begin{aligned}
N(y):=\{h \in C(J, E): h(t)= & S^{\prime}(t)\left[y_{0}-f(y)\right] \\
& \left.+\frac{d}{d t} \int_{0}^{t} S(t-s)\left[\mathcal{B} u_{y}(s)+v(s)\right] d s: v \in S_{F, y}\right\}
\end{aligned}
$$

has a fixed point.

STEP 1. $N(y)$ is convex for each $y \in C(J, E)$.

This is obvious, since $F$ has convex values.

STEP 2. $N$ maps bounded sets into bounded sets.

Indeed, if $h \in N(y)$ and $y \in B_{q}$, then there exists $v \in S_{F, y}$ such that for each $t \in J$ we have

$$
h(t)=S^{\prime}(t)\left[y_{0}-f(y)\right]+\frac{d}{d t} \int_{0}^{t} S(t-s)\left[\mathcal{B} u_{y}(s)+v(s)\right] d s .
$$

Observe that

$$
\begin{aligned}
\left|u_{y}(t)\right| & \leq M_{2}\left[\left|y_{1}\right|+G+M e^{\omega t}\left(\left|y_{0}\right|+G\right)+M e^{\omega t} \int_{0}^{t} e^{-\omega s}|v(s)| d s\right] \\
& \leq M_{2}\left[\left|y_{1}\right|+G+M^{*}\left(\left|y_{0}\right|+G\right)+M^{*} \int_{0}^{t} e^{-\omega s} h_{q}(s) d s\right]:=H^{*}
\end{aligned}
$$

with $h_{q}$ as in Definition 2.3 and $M^{*}=e^{\omega b}$ if $\omega>0$ or $M^{*}=1$ if $\omega \leq 0$. Then

$$
\|h\| \leq M^{*}\left[\left|y_{0}\right|+G\right]+M^{*} \int_{0}^{b} e^{-\omega s} h_{q}(s) d s+b M^{*} M_{1} M_{2} H^{*} .
$$

STEP 3. $N$ sends bounded sets into equicontinuous sets.

Let $h \in N(y)$ for $y \in B_{q}$. Let $\varepsilon>0$ and $\tau_{1}, \tau_{2} \in J$ with $\tau_{2}>\tau_{1}$. 
CASE 1. If $\tau_{1}>\varepsilon$ then

$$
\begin{aligned}
\mid h\left(\tau_{2}\right)- & h\left(\tau_{1}\right)|\leq|\left[S^{\prime}\left(\tau_{2}\right)-S^{\prime}\left(\tau_{1}\right)\right]\left[y_{0}-f(y)\right] \mid \\
& +\left|\lim _{\lambda \rightarrow \infty} \int_{0}^{\tau_{1}-\varepsilon}\left[S^{\prime}\left(\tau_{2}-s\right)-S^{\prime}\left(\tau_{1}-s\right)\right] B_{\lambda}\left[\mathcal{B} u_{y}(s)+v(s)\right] d s\right| \\
& +\left|\lim _{\lambda \rightarrow \infty} \int_{\tau_{1}-\varepsilon}^{\tau_{1}}\left[S^{\prime}\left(\tau_{2}-s\right)-S^{\prime}\left(\tau_{1}-s\right)\right] B_{\lambda}\left[\mathcal{B} u_{y}(s)+v(s)\right] d s\right| \\
& +\left|\lim _{\lambda \rightarrow \infty} \int_{\tau_{1}}^{\tau_{2}} S^{\prime}\left(\tau_{2}-s\right) B_{\lambda}\left[\mathcal{B} u_{y}(s)+v(s)\right] d s\right| \\
\leq & \left|\left(S^{\prime}\left(\tau_{2}\right)-S^{\prime}\left(\tau_{1}\right)\right) y_{0}\right| \\
& +M\left\|S^{\prime}\left(\tau_{2}-\tau_{1}+\varepsilon\right)-S^{\prime}(\varepsilon)\right\|_{B(E)}\left|f\left(B_{q}\right)\right| \\
& +M^{*}\left\|S^{\prime}\left(\tau_{2}-\tau_{1}+\varepsilon\right)-S^{\prime}(\varepsilon)\right\|_{B(E)} \int_{0}^{\tau_{1}-\varepsilon} e^{-\omega s}\left[M_{1} H^{*}+h_{q}(s)\right] d s \\
& +2 M^{*} \int_{\tau_{1}-\varepsilon}^{\tau_{1}} e^{-\omega s}\left[M_{1} H^{*}+h_{q}(s)\right] d s+M^{*} \int_{\tau_{1}}^{\tau_{2}} e^{-\omega s}\left[M_{1} H^{*}+h_{q}(s)\right] d s .
\end{aligned}
$$

CASE 2. Let $\tau_{1} \leq \varepsilon$. For $\tau_{2}-\tau_{1}<\varepsilon$ we get

$$
\begin{aligned}
\left|h\left(\tau_{2}\right)-h\left(\tau_{1}\right)\right| \leq & \left|\left(S^{\prime}\left(\tau_{2}\right)-S^{\prime}\left(\tau_{1}\right)\right) y_{0}\right|+M\left|S^{\prime}\left(\tau_{2}-\tau_{1}\right) f(y)-f(y)\right| \\
& +M^{*} \int_{0}^{2 \varepsilon} e^{-\omega s}\left[M_{1} H^{*}+h_{q}(s)\right] d s \\
& +M^{*} \int_{0}^{\varepsilon} e^{-\omega s}\left[M_{1} H^{*}+h_{q}(s)\right] d s .
\end{aligned}
$$

Now equicontinuity follows as in Theorem 3.1 with (5.2.5) replacing (3.1.6).

Fix $0<t \leq b$ and $0<\varepsilon<t$. For $y \in B_{q}$ and $v \in S_{F, y}$ we define

$$
\begin{aligned}
h_{\varepsilon}(t)= & S^{\prime}(t)\left[y_{0}-f(y)\right]+\lim _{\lambda \rightarrow \infty} \int_{0}^{t-\varepsilon} S^{\prime}(t-s) B_{\lambda}\left[\mathcal{B} u_{y}(s)+v(s)\right] d s \\
= & S^{\prime}(t)\left[y_{0}-f(y)\right] \\
& +S^{\prime}(\varepsilon) \lim _{\lambda \rightarrow \infty} \int_{0}^{t-\varepsilon} S^{\prime}(t-s-\varepsilon) B_{\lambda}\left[\mathcal{B} u_{y}(s)+v(s)\right] d s
\end{aligned}
$$

Note that

$$
\left\{\lim _{\lambda \rightarrow \infty} \int_{0}^{t-\varepsilon} S^{\prime}(t-s-\varepsilon) B_{\lambda}\left[\mathcal{B} u_{y}(s)+v(s)\right] d s: y \in B_{q} \text { and } v \in S_{F, y}\right\}
$$


is a bounded set since

$$
\begin{aligned}
&\left|\lim _{\lambda \rightarrow \infty} \int_{0}^{t-\varepsilon} S^{\prime}(t-s-\varepsilon) B_{\lambda}\left[\mathcal{B} u_{y}(s)+v(s)\right] d s\right| \\
& \leq M^{*} \int_{0}^{t-\varepsilon} e^{-\omega s}\left[M_{1} H^{*}+h_{q}(s)\right] d s .
\end{aligned}
$$

Since $S^{\prime}(t)$ is a compact operator for $t>0$, the set $Y_{\varepsilon}(t)=\left\{h_{\varepsilon}(t): y \in B_{q}\right.$ and $\left.v \in S_{F, y}\right\}$ is relatively compact in $E$ for every $0<\varepsilon<t$. Moreover for $h=h_{0}$,

$$
\left|h(t)-h_{\varepsilon}(t)\right| \leq M \int_{t-\varepsilon}^{t} e^{-\omega s}\left[M_{1} H^{*}+h_{q}(s)\right] d s .
$$

Therefore, $Y(t)=\left\{h(t): y \in B_{q}\right.$ and $\left.v \in S_{F, y}\right\}$ is totally bounded and hence relatively compact in $E$.

As a consequence of Steps 2, 3 and the Arzelà-Ascoli theorem we can conclude that $N: C(J, E) \rightarrow \mathcal{P}(C(J, E))$ is completely continuous.

STEP 4. $N$ has closed graph.

Let $y_{n} \rightarrow y_{*}, h_{n} \in N\left(y_{n}\right)$ and $h_{n} \rightarrow h_{*}$. Then there exists $v_{n} \in S_{F, y_{n}}$ such that

$$
h_{n}(t)=S^{\prime}(t)\left[y_{0}-f\left(y_{n}\right)\right]+\lim _{\lambda \rightarrow \infty} \int_{0}^{t} S^{\prime}(t-s) B_{\lambda}\left[\mathcal{B} u_{y_{n}}(s)+v_{n}(s)\right] d s, \quad t \in J
$$

We must prove that there exists $v_{*} \in S_{F, y_{*}}$ such that

$$
h_{*}(t)=S^{\prime}(t)\left[y_{0}-f\left(y_{*}\right)\right]+\lim _{\lambda \rightarrow \infty} \int_{0}^{t} S^{\prime}(t-s) B_{\lambda}\left[\mathcal{B} u_{y_{*}}(s)+v_{*}(s)\right] d s, \quad t \in J
$$

We have

$$
\begin{aligned}
& \|\left(h_{n}-S^{\prime}(t)\left[y_{0}-f\left(y_{n}\right)\right]-\lim _{\lambda \rightarrow \infty} \int_{0}^{t} S^{\prime}(t-s) B_{\lambda} \mathcal{B} u_{y_{n}}(s) d s\right) \\
& \quad-\left(h_{*}-S^{\prime}(t)\left[y_{0}-f\left(y_{*}\right)\right]-\lim _{\lambda \rightarrow \infty} \int_{0}^{t} S^{\prime}(t-s) B_{\lambda} \mathcal{B} u_{y_{*}}(s) d s\right) \| \rightarrow 0
\end{aligned}
$$

as $n \rightarrow \infty$. Consider the continuous linear operator $\Gamma: L^{1}(J, E) \rightarrow C(J, E)$ defined by

$$
(\Gamma v)(t)=\lim _{\lambda \rightarrow \infty} \int_{0}^{t} S^{\prime}(t-s) B_{\lambda} v(s) d s .
$$

It follows that $\Gamma \circ S_{F}$ has closed graph ([17]). Moreover, 


$$
h_{n}(t)-S^{\prime}(t)\left[y_{0}-f\left(y_{n}\right)\right]-\lim _{\lambda \rightarrow \infty} \int_{0}^{t} S^{\prime}(t-s) B_{\lambda} \mathcal{B} u_{y_{n}}(s) d s \in \Gamma\left(S_{F, y_{n}}\right) .
$$

Since $y_{n} \rightarrow y_{*}$, it follows that

$$
\begin{aligned}
h_{*}(t)-S^{\prime}(t)\left[y_{0}-f\left(y_{*}\right)\right]-\lim _{\lambda \rightarrow \infty} \int_{0}^{t} S^{\prime}(t-s) B_{\lambda} \mathcal{B} u_{y_{*}}(s) d s & \\
& =\lim _{\lambda \rightarrow \infty} \int_{0}^{t} S^{\prime}(t-s) B_{\lambda} v_{*}(s) d s
\end{aligned}
$$

for some $v_{*} \in S_{F, y_{*}}$.

STEP 5. There exists an open set $U \subseteq C(J, E)$ with $y \notin \sigma N y$ for all $\sigma \in(0,1)$ and $y \in \partial U$.

Let $\sigma \in(0,1)$ and assume $y \in \sigma N(y)$. Then

$$
y(t)=\sigma S^{\prime}(t)\left[y_{0}-f(y)\right]+\sigma \lim _{\lambda \rightarrow \infty} \int_{0}^{t} S^{\prime}(t-s) B_{\lambda}\left[\mathcal{B} u_{y}(s)+v(s)\right] d s .
$$

Thus

$$
\begin{aligned}
|y(t)| \leq & M e^{\omega t}\left[\left|y_{0}\right|+G\right]+M e^{\omega t} \int_{0}^{t} e^{-\omega s} p(s) \psi(\|y\|) d s+M e^{\omega t} \int_{0}^{t}\left|\mathcal{B} u_{y}(s)\right| d s \\
\leq & M^{*}\left[\left|y_{0}\right|+G\right]+M^{*} \int_{0}^{t} e^{-\omega s} p(s) \psi(\|y\|) d s \\
& +b M^{*} M_{1} M_{2}\left(\left|y_{1}\right|+G+M^{*}\left(\left|y_{0}\right|+G\right)+M^{*} \int_{0}^{b} e^{-\omega s} p(s) \psi(\|y\|) d s\right) \\
\leq & Z_{2}+M^{*} \int_{0}^{b} e^{-\omega s} p(s) \psi(\|y\|) d s+Z_{1} M^{*} \int_{0}^{b} e^{-\omega s} p(s) \psi(\|y\|) d s .
\end{aligned}
$$

Consequently,

$$
\frac{\|y\|}{Z_{2}+M^{*}\left(1+Z_{1}\right) \psi(\|y\|) \int_{0}^{b} p(s) d s} \leq 1
$$

Then by (5.2.5), there exists $M_{*}$ such that $\|y\| \neq M_{*}$. Set $U=\{y \in C(J, E)$ : $\left.\|y\|<M_{*}\right\}$ and conclude as before.

We also state without proof a result concerning the lower semicontinuous case for nondensely defined operators.

THEOREM 5.3. Assume that (3.1.3), (3.1.4), (3.5.1), (3.5.2), (5.2.1)-(5.2.6) are satisfied. Then the problem (1.1)-(1.2) is nonlocally controllable on J. 
6. Controllability for second order semilinear differential inclusions with nonlocal conditions. In this section we study the problem $(1.3)-(1.4)$.

Definition 6.1. A function $y \in C(J, E)$ is said to be a mild solution of $(1.3)-(1.4)$ if $y(0)+f(y)=y_{0}, y^{\prime}(0)+f_{1}(y)=\eta$ and there exists $v \in L^{1}(J, E)$ such that $v(t) \in F(t, y(t))$ a.e. on $J$ and

$$
y(t)=C(t)\left[y_{0}-f(y)\right]+S(t)\left[\eta-f_{1}(y)\right]+\int_{0}^{t} S(t-s)[\mathcal{B} u(s)+v(s)] d s .
$$

Definition 6.2. The system (1.3)-(1.4) is said to be nonlocally controllable on the interval $J$ if for every $y_{0}, \eta, y_{1} \in E$ there exists a control $u \in L^{2}(J, U)$ such that there exists a mild solution $y(t)$ of (1.3)-(1.4) satisfying $y(b)+f(y)=y_{1}$.

Theorem 6.1. Let $F: J \times E \rightarrow \mathcal{P}_{\mathrm{c}, \mathrm{cp}}(E)$. Assume (3.1.1) and the following conditions:

(6.1.1) $f, f_{1}: C(J, E) \rightarrow E$ are continuous and completely continuous and there exist constants $G, G_{1}>0$ such that $|f(y)| \leq G$ and $\left|f_{1}(y)\right| \leq G_{1}$ for all $y \in C(J, E)$;

(6.1.2) $A: D(A) \subset E \rightarrow E$ is the infinitesimal generator of a strongly continuous cosine family $\{C(t): t \in J\}$, and there exist constants $N_{1}, N_{2} \geq 1$ such that $\|C(t)\|_{B(E)} \leq N_{1},\|S(t)\|_{B(E)} \leq N_{2}$ for all $t \in \mathbb{R}$

(6.1.3) $\mathcal{B}$ is a continuous operator from $U$ to $E$ and the linear operator $W:$ $L^{2}(J, U) \rightarrow E$, defined by

$$
W u=\int_{0}^{b} S(b-s) \mathcal{B} u(s) d s
$$

has a bounded inverse $W^{-1}: E \rightarrow L^{2}(J, U)$ such that $\|\mathcal{B}\| \leq M_{1}$ and $\left\|W^{-1}\right\| \leq M_{2}$ for some positive constants $M_{1}, M_{2}$;

(6.1.4) for each bounded $Q \subseteq C(J, E)$, and $t \in J$ the set

$$
\begin{aligned}
\left\{C(t)\left[y_{0}-f(y)\right]+S(t)[\eta\right. & \left.-f_{1}(y)\right] \\
& \left.+\int_{0}^{t} S(t-s)\left[\mathcal{B} u_{y}(s)+v(s)\right] d s: v \in S_{F, Q}\right\}
\end{aligned}
$$

is relatively compact in $E$, where $y \in B$ and $S_{F, Q}=\bigcup\left\{S_{F, y}: y \in Q\right\}$ and

$$
\begin{aligned}
u_{y}(t)= & W^{-1}\left[y_{1}-f(y)-C(b)\left(y_{0}-f(y)\right)-S(b)\left(\eta-f_{1}(y)\right)\right. \\
& \left.-\int_{0}^{b} S(b-s) v(s) d s\right](t), \quad v \in S_{F, y} ;
\end{aligned}
$$


(6.1.5) there exists a continuous nondecreasing function $\psi:[0, \infty) \rightarrow(0, \infty)$ and $p_{1} \in L^{1}\left(J, \mathbb{R}_{+}\right)$such that

$$
\|F(t, u)\| \leq p(t) \psi(|u|) \quad \text { for }(t, u) \in J \times E
$$

and there exists a constant $M_{* *}>0$ with

$$
\frac{M_{* *}}{\Lambda_{1}+\left(\Lambda_{2}+N_{2}\right) \psi\left(M_{* *}\right) \int_{0}^{b} p_{1}(s) d s}>1
$$

where

$$
\begin{aligned}
\Lambda_{1}= & N_{1}\left|y_{0}\right|+N_{1} G+N_{2}|\eta|+N_{2} G_{1} \\
& +b N_{2} M_{1} M_{2}\left[\left|y_{1}\right|+G+N_{1}\left|y_{0}\right|+N_{1} G+N_{2}|\eta|+N_{2} G_{1}\right], \\
\Lambda_{2}= & b N_{2}^{2} M_{1} M_{2} ;
\end{aligned}
$$

(6.1.6) given $\varepsilon>0$, for any bounded subset $D$ of $C(J, E)$ there exists a $\delta>0$ with $\left.\mid\left[C\left(\tau_{2}\right)-C\left(\tau_{1}\right)\right] f(y)\right] \mid<\varepsilon$ for all $y \in D$ and $\tau_{1}, \tau_{2} \in[0, \delta]$.

Then the problem (1.3)-(1.4) is nonlocally controllable on $J$.

Proof. Using hypothesis (6.1.3), for any $y \in C(J, E)$ define the control

$$
\begin{aligned}
u_{y}(t)= & W^{-1}\left[y_{1}-f(y)-C(b)\left(y_{0}-f(y)\right)-S(b)\left(\eta-f_{1}(y)\right)\right. \\
& \left.-\int_{0}^{b} S(b-s) v(s) d s\right](t),
\end{aligned}
$$

where $v \in S_{F, y}$. We will show that the operator $\bar{N}: C(J, E) \rightarrow \mathcal{P}(C(J, E))$ defined by

$$
\begin{aligned}
\bar{N}(y):=\{h \in C(J, E): h(t)= & C(t)\left[y_{0}-f(y)\right]+S(t)\left[\eta-f_{1}(y)\right] \\
& \left.+\int_{0}^{t} S(t-s)\left[\mathcal{B} u_{y}(s)+v(s)\right] d s: v \in S_{F, y}\right\}
\end{aligned}
$$

has a fixed point.

STEP 1. $\bar{N}(y)$ is convex for each $y \in C(J, E)$.

SteP 2. $\bar{N}$ maps bounded sets into bounded sets.

Indeed, if $h \in \bar{N}(y)$ and $y \in B_{q}$, then there exists $v \in S_{F, y}$ such that for each $t \in J$,

$$
h(t)=C(t)\left[y_{0}-f(y)\right]+S(t)\left[\eta-f_{1}(y)\right]+\int_{0}^{t} S(t-s)\left[\mathcal{B} u_{y}(s)+v(s)\right] d s .
$$

Observe that

$$
\left|u_{y}(t)\right| \leq M_{2}\left[\left|y_{1}\right|+G+N_{1}\left|y_{0}\right|+N_{1} G+N_{2}|\eta|+N_{2} G_{1}+N_{2}\left\|h_{q}\right\|_{L^{1}}\right]:=H_{2}
$$

with $h_{q}$ as in Definition 2.3. Thus for each $t \in J$ we get 


$$
\begin{aligned}
|h(t)| & \leq N_{1}\left|y_{0}\right|+N_{1} G+N_{2}|\eta|+N_{2} G_{1}+b N_{2} M_{1} H_{2}+N_{2} \int_{0}^{t}|v(s)| d s \\
& \leq N_{1}\left|y_{0}\right|+N_{1} G+N_{2}|\eta|+N_{2} G_{1}+b N_{2} M_{1} H_{2}+N_{2}\left\|h_{q}\right\|_{L^{1}} .
\end{aligned}
$$

SteP 3. $\bar{N}$ sends bounded sets into equicontinuous sets.

Fix $\tau_{1}, \tau_{2} \in J$ with $\tau_{2}>\tau_{1}$. For $y \in B_{q}$, using Proposition 2.2 we have

$$
\begin{aligned}
\left|h\left(\tau_{2}\right)-h\left(\tau_{1}\right)\right| \leq & \left.\left|\left[C\left(\tau_{2}\right)-C\left(\tau_{1}\right)\right] y_{0}\right|+\mid\left[C\left(\tau_{2}\right)-C\left(\tau_{1}\right)\right] f(y)\right] \mid \\
& \left.+\left|\left[S\left(\tau_{2}\right)-S\left(\tau_{1}\right)\right] \eta\right|+\mid\left[S\left(\tau_{2}\right)-S\left(\tau_{1}\right)\right] f_{1}(y)\right] \mid \\
& +\int_{0}^{\tau_{1}}\left|\left[S\left(\tau_{2}-s\right)-S\left(\tau_{1}-s\right)\right]\left[\mathcal{B} u_{y}(s)+v(s)\right]\right| d s \\
& +\int_{\tau_{1}}^{\tau_{2}}\left|S\left(\tau_{2}-s\right)\left[\mathcal{B} u_{y}(s)+v(s)\right]\right| d s \\
\leq & \left.\left|\left[C\left(\tau_{2}\right)-C\left(\tau_{1}\right)\right] y_{0}\right|+\mid\left[C\left(\tau_{2}\right)-C\left(\tau_{1}\right)\right] f(y)\right] \mid \\
& +\left|\left[S\left(\tau_{2}\right)-S\left(\tau_{1}\right)\right] \eta\right|+G_{1} N_{2} \int_{\tau_{1}} e^{\omega x} d x \\
& +\int_{0}^{\tau_{1} \tau_{2}-s} \int_{\tau_{1}-s}^{\omega x} d x\left|\mathcal{B} u_{y}(s)+v(s)\right| d s \\
& +N_{2} \int_{\tau_{1}}^{\tau_{2}}\left[M_{1} H_{2}+h_{q}(s)\right] d s \\
\leq & \left.\left|\left[C\left(\tau_{2}\right)-C\left(\tau_{1}\right)\right] y_{0}\right|+\mid\left[C\left(\tau_{2}\right)-C\left(\tau_{1}\right)\right] f(y)\right] \mid \\
& +\left|\left[S\left(\tau_{2}\right)-S\left(\tau_{1}\right)\right] \eta\right|+G_{1} N_{2} e^{\omega b}\left(\tau_{2}-\tau_{1}\right) \\
& +e^{\omega b}\left(\tau_{2}-\tau_{1}\right) \int_{1}\left[M_{1} H_{2}+h_{q}(s)\right] d s \\
& +N_{2} \int_{\tau_{1}}^{\tau_{2}}\left[M_{1} H_{2}+h_{q}(s)\right] d s . \\
& \\
& \\
& \\
& \\
&
\end{aligned}
$$

As a consequence of Steps 2, 3, (6.1.4) and the Arzelà-Ascoli theorem, $\bar{N}$ is completely continuous.

STEP 4. $\bar{N}$ has closed graph.

Let $y_{n} \rightarrow y_{*}, h_{n} \in N\left(y_{n}\right)$ and $h_{n} \rightarrow h_{*}$. Then there exists $v_{n} \in S_{F, y_{n}}$ such that

$$
\begin{aligned}
h_{n}(t)= & C(t)\left[y_{0}-f\left(y_{n}\right)\right]+S(t)\left[\eta-f_{1}\left(y_{n}\right)\right] \\
& +\int_{0}^{t} S(t-s)\left[\mathcal{B} u_{y_{n}}(s)+v_{n}(s)\right] d s, \quad t \in J,
\end{aligned}
$$


where $u_{y_{n}}(t)$ is as in (6.1.4). We must prove that there exists $v_{*} \in S_{F, y_{*}}$ such that

$$
h_{*}(t)=C(t)\left[y_{0}-f\left(y_{*}\right)\right]+S(t)\left[\eta-f_{1}\left(y_{*}\right)\right]+\int_{0}^{t} S(t-s)\left[\mathcal{B} u_{y_{*}}(s)+v_{*}(s)\right] d s
$$

for $t \in J$. Set

$$
\bar{u}_{y}(t)=W^{-1}\left[y_{1}-f(y)-C(b)\left(y_{0}-f(y)\right)-S(b)\left(\eta-f_{1}(y)\right)\right] .
$$

Since $f$ and $W^{-1}$ are continuous, $\bar{u}_{y_{n}}(t) \rightarrow \bar{u}_{y^{*}}(t)$, for $t \in J$.

We have

$$
\begin{aligned}
& \left.\|\left(h_{n}-C(t)\left[y_{0}-f\left(y_{n}\right)\right]-S(b)\left[\eta-f_{1}\left(y_{n}\right)\right]\right)-\int_{0}^{t} T(t-s) \mathcal{B} \bar{u}_{y_{n}}(s) d s\right) \\
& -\left(h_{*}-T(t)\left[y_{0}-f\left(y_{*}\right)\right]-S(b)\left[\eta-f_{1}\left(y_{*}\right)\right]-\int_{0}^{t} T(t-s) \mathcal{B} \bar{u}_{y_{*}}(s) d s\right) \| \rightarrow 0
\end{aligned}
$$

as $n \rightarrow \infty$. Consider the operator $\Gamma: L^{1}(J, E) \rightarrow C(J, E)$ defined by

$$
(\Gamma v)(t)=\int_{0}^{t} S(t-s)\left[\mathcal{B} W^{-1}\left(\int_{0}^{b} S(b-\tau) v(\tau) d \tau\right)(s)+v(s)\right] d s .
$$

Clearly, $\Gamma$ is linear and continuous. Indeed,

$$
\|\Gamma v\| \leq b N_{2}\left(b N_{2} M_{1} M_{2}+1\right)\|v\|_{L^{1}} .
$$

It follows that $\Gamma \circ S_{F}$ has closed graph ([17]). Moreover

$$
h_{n}(t)-C(t)\left[y_{0}-f\left(y_{n}\right)\right]-S(t)\left[\eta-f_{1}\left(y_{n}\right)\right]-\int_{0}^{t} T(t-s) \mathcal{B} \bar{u}_{y_{n}}(s) \in \Gamma\left(S_{F, y_{n}}\right) .
$$

Since $y_{n} \rightarrow y_{*}$, it follows that

$$
\begin{aligned}
h_{*}(t)= & C(t)\left[y_{0}-f\left(y_{*}\right)\right]+S(t)\left[\eta-f_{1}\left(y_{*}\right)\right] \\
& +\int_{0}^{t} T(t-s)\left[\mathcal{B} u_{y_{*}}(s)+v_{*}(s)\right] d s, \quad t \in J,
\end{aligned}
$$

for some $v_{*} \in S_{F, y_{*}}$.

STEP 5. There exists an open set $U \subseteq C(J, E)$ with $y \notin \lambda \bar{N} y$ for any $\lambda \in(0,1)$ and $y \in \partial U$.

Let $\lambda \in(0,1)$ and assume $y \in \lambda \bar{N}(y)$. Then for $t \in J$,

$$
\begin{aligned}
|y(t)| \leq & N_{1}\left|y_{0}\right|+N_{1} G+N_{2}|\eta|+N_{2} G_{1} \\
& +N_{2} \int_{0}^{t}\left|\mathcal{B} u_{y}(s)\right| d s+N_{2} \int_{0}^{t} p(s) \psi(\|y\|) d s
\end{aligned}
$$




$$
\begin{aligned}
\leq & N_{1}\left|y_{0}\right|+N_{1} G+N_{2}|\eta|+N_{2} G_{1} \\
& +b N_{2} M_{1} M_{2}\left[\left|y_{1}\right|+G+N_{1}\left|y_{0}\right|+N_{1} G+N_{2}|\eta|+N_{2} G_{1}\right. \\
& \left.+N_{2} \int_{0}^{b} p(s) \psi(\|y\|) d s\right]+N_{2} \int_{0}^{t} p(s) \psi(\|y\|) d s \\
\leq & \Lambda_{1}+\Lambda_{2} \psi(\|y\|) \int_{0}^{b} p(s) d s+N_{2} \psi(\|y\|) \int_{0}^{t} p(s) d s .
\end{aligned}
$$

Consequently,

$$
\frac{\|y\|}{\Lambda_{1}+\left(\Lambda_{2}+N_{2}\right) \psi(\|y\|) \int_{0}^{b} p(s) d s} \leq 1 .
$$

Then by (6.1.5), there exists $M_{* *}$ such that $\|y\| \neq M_{* *}$. Set

$$
U=\left\{y \in C(J, E):\|y\|<M_{* *}\right\}
$$

and finish the proof as before.

REMARK 6.1. If $E$ is finite-dimensional then (6.1.4) is satisfied. Condition (6.1.4) is only needed to guarantee that $\left\{\bar{N} y(t): y \in B_{q}\right\}$ is relatively compact for each $t \in J$ so the Arzelà-Ascoli theorem can be applied. Conditions of the type (6.1.4) have been discussed extensively in the literature.

In the next result we give the analogue of Theorem 3.4 for the problem (1.3)-(1.4). The proof follows closely the ideas of Theorem 3.4 and is omitted.

Theorem 6.2. Suppose (3.1.1), (3.4.1), (6.1.2), (6.1.3), (6.1.4) and (6.1.6) hold. In addition assume that:

(6.2.1) $f_{1}: C(J, E) \rightarrow E$ is continuous and completely continuous and there exists a continuous nondecreasing function $\psi_{1}:[0, \infty) \rightarrow[0, \infty)$ with

$$
\left|f_{1}(y)\right| \leq \psi_{1}(\|y\|) \quad \text { for } y \in C(J, E)
$$

(6.2.2) there exists a continuous nondecreasing function $g_{1}:[0, \infty) \rightarrow(0, \infty)$ and $p_{1} \in L^{1}\left(J, \mathbb{R}_{+}\right)$such that

$$
\|F(t, u)\| \leq p_{1}(t) g_{1}(|u|) \quad \text { for }(t, u) \in J \times E
$$

and there exists a constant $M_{* *}^{\prime}>0$ with

$$
\begin{aligned}
& \frac{M_{* *}^{\prime}}{\left.\Lambda_{1}^{\prime}+\left[\Lambda_{2}^{\prime}+N_{1} \Lambda_{3}^{\prime}\right)\right] \psi\left(M_{* *}^{\prime}\right)+N_{2} \Lambda_{3}^{\prime} \psi_{1}\left(M_{* *}^{\prime}\right)+N_{2} \Lambda_{3}^{\prime} g_{1}\left(M_{* *}^{\prime}\right) \int_{0}^{b} p_{1}(s) d s}>1, \\
& \text { where } \\
& \Lambda_{1}^{\prime}=N_{1}\left|y_{0}\right|+N_{2}|\eta|+b N_{2} M_{1} M_{2}\left[\left|y_{1}\right|+N_{1}\left|y_{0}\right|+N_{2}|\eta|\right] \\
& \Lambda_{2}^{\prime}=b N_{2} M_{1} M_{2} \\
& \Lambda_{3}^{\prime}=1+\Lambda_{2}^{\prime} .
\end{aligned}
$$

Then the problem (1.3)-(1.4) is nonlocally controllable on $J$. 
If we have at most linear growth then we have the following

Theorem 6.3. Assume that (3.1.1), (6.1.1)-(6.1.4) and (6.1.6) hold. In addition suppose that:

(6.3.1) there exists $p \in L^{1}\left(J, \mathbb{R}_{+}\right)$and positive constants $A_{1}$ and $B_{1}$ such that

$$
\|F(t, u)\| \leq p(t)\left[A_{1}|u|+B_{1}\right] \quad \text { for }(t, u) \in J \times E ;
$$

(6.3.2) $A_{1} \Lambda_{2} e^{A_{1} N_{2} \int_{0}^{b} p(s) d s} \int_{0}^{b} p(t) e^{-A_{1} N_{2} \int_{0}^{t} p(s) d s} d t<1, \quad \Lambda_{2}=b^{3} N_{2}^{2} M_{1} M_{2}$.

Then the problem (1.3)-(1.4) is nonlocally controllable on $J$.

Proof. Let $\lambda \in(0,1)$ and assume $y \in \lambda \bar{N}(y)$ where $\bar{N}$ is as in Theorem 6.1. For each $t \in J$ we have

$$
|y(t)| \leq \Lambda_{1}+\Lambda_{2} \int_{0}^{b} p(s)\left[A_{1}|y(s)|+B_{1}\right] d s+N_{2} \int_{0}^{t} p(s)\left[A_{1}|y(s)|+B_{1}\right] d s .
$$

Let $v(t)=\int_{0}^{t} p(s)\left[A_{1}|y(s)|+B_{1}\right] d s$. Then $v(0)=0$ and

$$
\begin{aligned}
v^{\prime}(t) & =p(t)\left[A_{1}|y(t)|+B_{1}\right] \\
& \leq p(t)\left\{A_{1}\left[\Lambda_{1}+\Lambda_{2} v(b)+N_{2} v(t)\right]+B_{1}\right\} \\
& \leq p(t) A_{1} N_{2} v(t)+p(t) A_{1} \Lambda_{2} v(b)+p(t)\left(A_{1} \Lambda_{1}+B_{1}\right) .
\end{aligned}
$$

Multiply both sides by $e^{-A_{1} N_{2} \int_{0}^{t} p(s) d s}$ to get

$$
\begin{aligned}
\left(v(t) e^{-A_{1} N_{2} \int_{0}^{t} p(s) d s}\right)^{\prime} \leq & A_{1} \Lambda_{2} v(b) p(t) e^{-A_{1} N_{2} \int_{0}^{t} p(s) d s} \\
& +p(t)\left(A_{1} \Lambda_{1}+B_{1}\right) e^{-A_{1} N_{2} \int_{0}^{t} p(s) d s} .
\end{aligned}
$$

Integrating from 0 to $b$ we get

$$
\begin{aligned}
v(b) e^{-A_{1} N_{2} \int_{0}^{b} p(s) d s} \leq & A_{1} \Lambda_{2} v(b) \int_{0}^{b} p(t) e^{-A_{1} N_{2} \int_{0}^{t} p(s) d s} d t \\
& +\left(A_{1} \Lambda_{1}+B_{1}\right) \int_{0}^{b} p(t) e^{-A_{1} N_{2} \int_{0}^{t} p(s) d s} d t
\end{aligned}
$$

or

$$
v(b) \leq \frac{\left(A_{1} \Lambda_{1}+B_{1}\right) \int_{0}^{b} p(t) e^{-A_{1} N_{2} \int_{0}^{t} p(s) d s} d t}{e^{-A_{1} N_{2} \int_{0}^{b} p(s) d s}-A_{1} \Lambda_{2} \int_{0}^{b} p(t) e^{-A_{1} N_{2} \int_{0}^{t} p(s) d s} d t}=: K_{0}^{\prime} .
$$

Thus $\|v\| \leq K_{0}^{\prime}$, so $\|y\| \leq \Lambda_{1}+\left(\Lambda_{2}+N_{2}\right) K_{0}^{\prime} \equiv K_{1}^{\prime}$. Set $M_{*}=K_{1}^{\prime}+1$ and apply the nonlinear alternative as in Theorem 6.1.

For the lower semicontinuous case we state without proof the following result. 
Theorem 6.4. Assume that (3.5.1), (3.5.2), (6.1.1)-(6.1.6) are satisfied. Then the problem (1.3)-(1.4) is nonlocally controllable on $J$.

REMARK 6.2. The above method can be used to enlarge the class of second order problems, for example to nonlocal quasilinear damped differential inclusions of the form

$$
\begin{gathered}
y^{\prime \prime}(t)-B y^{\prime}(t) \in A y(t)+F(t, y(t))+\mathcal{B} u(t), \quad t \in J:=[0, b], \\
y(0)+f(y)=y_{0}, \quad y^{\prime}(0)+f_{1}(y)=\eta
\end{gathered}
$$

where $A, F, y_{0}, f, f_{1}, \mathcal{B}, u, \eta$ are as in problem (6.1)-(6.2) and $\mathcal{B}$ is a bounded linear operator in $E$.

\section{References}

[1] W. Arendt, Vector valued Laplace transforms and Cauchy problems, Israel J. Math. 59 (1987), 327-352.

[2] J.-P. Aubin and A. Cellina, Differential Inclusions, Springer, Birkhäuser, New York, 1984.

[3] M. Benchohra, L. Górniewicz and S. K. Ntouyas, Controllability of Some Nonlinear Systems in Banach Spaces, Paweł Włodkowic University College, Płock, 2003.

[4] A. Bressan and G. Colombo, Extensions and selections of maps with decomposable values, Studia Math. 90 (1988), 69-86.

[5] L. Byszewski, Theorems about the existence and uniqueness of solutions of a semilinear evolution nonlocal Cauchy problem, J. Math. Anal. Appl. 162 (1991), 494-505.

[6] -, Existence and uniqueness of a classical solution to a functional-differential abstract nonlocal Cauchy problem, J. Appl. Math. Stoch. Anal. 12 (1999), 91-97.

[7] G. Da Prato and E. Sinestrari, Differential operators with non-dense domains, Ann. Scuola Norm. Sup. Pisa Cl. Sci. 14 (1987), 285-344.

[8] K. Deimling, Multivalued Differential Equations, de Gruyter, Berlin, 1992.

[9] H. O. Fattorini, Second Order Linear Differential Equations in Banach Spaces, North-Holland Math. Stud. 108, North-Holland, Amsterdam, 1985.

[10] L. Górniewicz, Topological Fixed Point Theory of Multivalued Mappings, Math. Appl. 495, Kluwer, Dordrecht, 1999.

[11] L. Górniewicz, S. K. Ntouyas and D. O'Regan, Controllability of semilinear differential equations and inclusions via semigroup theory in Banach spaces, Rep. Math. Phys. 56 (2005), 437-470.

[12] J. A. Goldstein, Semigroups of Linear Operators and Applications, Oxford Univ. Press, New York, 1985.

[13] A. Granas and J. Dugundji, Fixed Point Theory, Springer, New York, 2003.

[14] S. Heikkila and V. Lakshmikantham, Monotone Iterative Techniques for Discontinuous Nonlinear Differential Equations, Dekker, New York, 1994.

[15] Sh. Hu and N. Papageorgiou, Handbook of Multivalued Analysis, Vol. I: Theory, Kluwer, Dordrecht, 1997.

[16] H. Kellerman and M. Hieber, Integrated semigroups, J. Funct. Anal. 84 (1989), $160-180$.

[17] A. Lasota and Z. Opial, An application of the Kakutani-Ky Fan theorem in the theory of ordinary differential equations, Bull. Acad. Polon. Sci. Sér. Sci. Math. Astronom. Phys. 13 (1965), 781-786. 
[18] A. Pazy, Semigroups of Linear Operators and Applications to Partial Differential Equations, Springer, New York, 1983.

[19] C. Travis and G. Webb, Existence and stability for partial functional differential equations, Trans. Amer. Math. Soc. 200 (1974), 395-418.

[20] - - - Cosine families and abstract nonlinear second order differential equations, Acta Math. Hungar. 32 (1978), 75-96.

[21] - - - An abstract second order semilinear Volterra integrodifferential equation, SIAM J. Math. Anal. 10 (1979), 412-424.

Faculty of Mathematics and Computer Science

Nicolaus Copernicus University

Chopina $12 / 18$

87-100 Torun, Poland

E-mail: gorn@mat.uni.torun.pl

Department of Mathematics

National University of Ireland

Galway, Ireland

E-mail: donal.oregan@unigalway.ie
Department of Mathematics University of Ioannina 45110 Ioannina, Greece E-mail: sntouyas@uoi.gr

Received 22.3.2006

and in final form 28.6.2006 